\title{
EQUIDISTRIBUTION FOR SEQUENCES OF LINE BUNDLES ON NORMAL KÄHLER SPACES
}

\author{
DAN COMAN, XIAONAN MA, AND GEORGE MARINESCU
}

\begin{abstract}
AвSTRACT. We study the asymptotics of Fubini-Study currents and zeros of random holomorphic sections associated to a sequence of singular Hermitian line bundles on a compact normal Kähler complex space.
\end{abstract}

\section{CONTENTS}

1. Introduction

2. Preliminaries

2.1. Plurisubharmonic functions and currents on analytic spaces

2.2. Singular Hermitian holomorphic line bundles on analytic spaces

2.3. Resolution of singularities

2.4. $L^{2}$-estimates for $\bar{\partial}$

2.5. Special weights of Hermitian metrics on reference covers

3. Proofs of Theorems 1.1, 1.2 and 1.3

4. Zeros of holomorphic sections and approximation results

5. Applications

5.1. Powers of a line bundle

5.2. Powers of ample line bundles

5.3. Tensor products of powers of several line bundles

5.4. Covering manifolds

References

\section{INTRODUCTION}

In this paper we continue the study of equidistribution of Fubini-Study currents and zeros of sequences of holomorphic sections of singular Hermitian holomorphic bundles

Date: January 1, 2016.

2010 Mathematics Subject Classification. Primary 32L10; Secondary 32A60, 32C20, 32U40, 81Q50.

Key words and phrases. Bergman kernel function, Fubini-Study current, singular Hermitian metric, compact normal Kähler complex space, zeros of random holomorphic sections.

D. Coman is partially supported by the NSF Grant DMS-1300157.

$\mathrm{X}$. Ma is partially supported by Institut Universitaire de France.

G. Marinescu is partially supported by DFG funded projects SFB/TR 12, MA 2469/2-2.

Funded through the Institutional Strategy of the University of Cologne within the German Excellence Initiative. 
started in [CM1, CM2, CM3]. We generalize our previous results in two directions. On the one hand, we allow the base space to be singular and work over Kähler spaces. On the other hand, we consider sequences $\left(L_{p}, h_{p}\right), p \geq 1$, of singular Hermitian holomorphic line bundles whose Chern curvature satisfy a natural growth condition, instead of sequences of powers $\left(L^{p}, h^{p}\right)$ of a fixed line bundle $(L, h)$.

Recall that by the results of [T1] (see also [MM1, Section 5.3]), if $(X, \omega)$ is a compact Kähler manifold whose Kähler form is integral and $(L, h)$ is a prequantum line bundle (i.e., the Chern curvature form $c_{1}(L, h)$ equals $\omega$ ), then the normalized Fubini-Study currents $\frac{1}{p} \gamma_{p}$ associated to $H^{0}\left(X, L^{p}\right)$ converge in the $\mathscr{C}^{2}$ topology to $\omega$. This result can be applied to describe the asymptotic distribution of zeros of random sequences of holomorphic sections. Indeed, it is shown in [SZ1] (see also [NV, DS1, SZ2, Sh, DMS]) that for almost all sequences $\left\{\sigma_{p} \in H^{0}\left(X, L^{p}\right)\right\}_{p \geq 1}$ the normalized zero-currents $\frac{1}{p}\left[\sigma_{p}=0\right]$ converge weakly to $\omega$ on $X$. This means that the Kähler form $\omega$ can be approximated by various algebraic/analytic objects in the semiclassical limit $p \rightarrow \infty$. Some important technical tools for higher dimension used in the these works were introduced in [FS]. We note that statistics of zeros of sections and hypersurfaces have been studied also in the context of real manifolds and real vector bundles, see e.g. [GW, NS].

In [CM1, CM2, CM3] we relaxed the smoothness condition on $\omega$ and assumed that $\omega$ is merely an integral Kähler current. Then there exists a holomorphic line bundle $(L, h)$ endowed with a singular Hermitian metric such that $c_{1}(L, h)$ equals $\omega$ as currents. We established the above approximation results in the sense of currents by working with the spaces of square integrable holomorphic sections $H_{(2)}^{0}\left(X, L^{p}\right)$. The setting in [CM1, CM2, CM3] allows to deal with several singular Kähler metrics such as singular metrics on big line bundles, metrics with Poincaré growth, Kähler-Einstein metrics singular along a divisor (e.g. good metrics in the sense of Mumford on toroidal compactifications of arithmetic quotiens [AMRT]) or metrics on orbifold line bundles.

We consider in this paper the following setting:

(A) $(X, \omega)$ is a compact (reduced) normal Kähler space of pure dimension $n, X_{\text {reg }}$ denotes the set of regular points of $X$, and $X_{\text {sing }}$ denotes the set of singular points of $X$.

(B) $\left(L_{p}, h_{p}\right), p \geq 1$, is a sequence of holomorphic line bundles on $X$ with singular Hermitian metrics $h_{p}$ whose curvature currents verify

$$
c_{1}\left(L_{p}, h_{p}\right) \geq a_{p} \omega \text { on } X \text {, where } a_{p}>0 \text { and } \lim _{p \rightarrow \infty} a_{p}=\infty .
$$

Let $A_{p}=\int_{X} c_{1}\left(L_{p}, h_{p}\right) \wedge \omega^{n-1}$. If $X_{\text {sing }} \neq \emptyset$ we assume moreover that

$$
\exists T_{0} \in \mathscr{T}(X) \text { such that } c_{1}\left(L_{p}, h_{p}\right) \leq A_{p} T_{0}, \forall p \geq 1 .
$$

Here we consider currents on the analytic space $X$ in the sense of [D3], and $\mathscr{T}(X)$ is the set of positive closed currents of bidegree $(1,1)$ on $X$ which have a local plurisubharmonic (psh) potential in the neighborhood of each point of $X$ (see Section 2). 
We let $H_{(2)}^{0}\left(X, L_{p}\right)$ be the Bergman space of $L^{2}$-holomorphic sections of $L_{p}$ relative to the metric $h_{p}$ and the volume form induced by $\omega$ on $X$,

$$
H_{(2)}^{0}\left(X, L_{p}\right)=\left\{S \in H^{0}\left(X, L_{p}\right):\|S\|_{p}^{2}:=\int_{X_{\mathrm{reg}}}|S|_{h_{p}}^{2} \frac{\omega^{n}}{n !}<\infty\right\},
$$

endowed with the obvious inner product. Furthermore, we denote by $P_{p}$, resp. $\gamma_{p}$, the Bergman kernel function, resp. the Fubini-Study current, of the space $H_{(2)}^{0}\left(X, L_{p}\right)$, which are defined as follows. For $p \geq 1$, let $S_{1}^{p}, \ldots, S_{d_{p}}^{p}$ be an orthonormal basis of $H_{(2)}^{0}\left(X, L_{p}\right)$. If $x \in X$ and $e_{p}$ is a local holomorphic frame of $L_{p}$ in a neighborhood $U_{p}$ of $x$ we write $S_{j}^{p}=s_{j}^{p} e_{p}$, where $s_{j}^{p} \in \mathcal{O}_{X}\left(U_{p}\right)$. Then

$$
P_{p}(x)=\sum_{j=1}^{d_{p}}\left|S_{j}^{p}(x)\right|_{h_{p}}^{2},\left.\quad \gamma_{p}\right|_{U_{p}}=\frac{1}{2} d d^{c} \log \left(\sum_{j=1}^{d_{p}}\left|s_{j}^{p}\right|^{2}\right),
$$

where $d^{c}=\frac{1}{2 \pi i}(\partial-\bar{\partial})$. Note that $P_{p}, \gamma_{p}$ are independent of the choice of basis.

Our main results are the following theorems:

Theorem 1.1. If $(X, \omega),\left(L_{p}, h_{p}\right), p \geq 1$, verify assumptions $(A)$-(B), then:

(i) $\frac{1}{A_{p}} \log P_{p} \rightarrow 0$ as $p \rightarrow \infty$, in $L^{1}\left(X, \omega^{n}\right)$.

(ii) $\frac{1}{A_{p}}\left(\gamma_{p}-c_{1}\left(L_{p}, h_{p}\right)\right) \rightarrow 0$ as $p \rightarrow \infty$, in the weak sense of currents on $X$.

Moreover, if $\frac{1}{A_{p}} c_{1}\left(L_{p}, h_{p}\right) \rightarrow T$ for some positive closed current $T$ of bidegree $(1,1)$ on $X$, then $\frac{1}{A_{p}} \gamma_{p} \rightarrow T$ as $p \rightarrow \infty$, in the weak sense of currents on $X$.

When $X$ is smooth we obtain:

Theorem 1.2. Let $(X, \omega)$ be a compact Kähler manifold of dimension $n$ and $\left(L_{p}, h_{p}\right), p \geq 1$, be a sequence of singular Hermitian holomorphic line bundles on $X$ which satisfy $c_{1}\left(L_{p}, h_{p}\right) \geq$ $a_{p} \omega$, where $a_{p}>0$ and $\lim _{p \rightarrow \infty} a_{p}=\infty$. If $A_{p}=\int_{X} c_{1}\left(L_{p}, h_{p}\right) \wedge \omega^{n-1}$ then $\frac{1}{A_{p}} \log P_{p} \rightarrow 0$ in $L^{1}\left(X, \omega^{n}\right)$ and $\frac{1}{A_{p}}\left(\gamma_{p}-c_{1}\left(L_{p}, h_{p}\right)\right) \rightarrow 0$ weakly on $X$, as $p \rightarrow \infty$.

If $\left(L_{p}, h_{p}\right)=\left(L^{p}, h^{p}\right)$, where $(L, h)$ is a fixed singular Hermitian holomorphic line bundle, we obtain analogues of the equidistributions results from [SZ1, DS1, CM1, CM2, CM3] for compact normal Kähler spaces. Note that in this case hypothesis (2) is automatically verified as $c_{1}\left(L^{p}, h^{p}\right)=p c_{1}(L, h)$, so we can take $A_{p}=p$ and $T_{0}=c_{1}(L, h)$.

In the case $\left(L_{p}, h_{p}\right)=\left(L^{p}, h^{p}\right)$, Theorem 1.1 gives semiclassical approximation results for integral Kähler currents. In order to deal with the non-integral case, we consider those currents $T$ which can be approximated by the curvatures of a sequence $\left(L_{p}, h_{p}\right)$, $p \geq 1$, of singular Hermitian holomorphic line bundles. Such a sequence can be thought as a "prequantization" of the non-integral positive closed $(1,1)$ current $T$. Theorems 1.1-1.2 and their consequences are a manifestation of the quantum ergodicity in this context. For details, see Sections 4, 5, and Theorem 4.7. Another interesting situation is when $L_{p}$ equals a product of tensor powers of several holomorphic line bundles, $L_{p}=$ $F_{1}^{m_{1, p}} \otimes \ldots \otimes F_{k}^{m_{k, p}}$, where $\left\{m_{j, p}\right\}_{p}, 1 \leq j \leq k$, are sequences in $\mathbb{N}$ such that $m_{j, p}=r_{j} p+o(p)$ as $p \rightarrow \infty$, where $r_{j}>0$ are given. This means that $\left(m_{1, p}, \ldots, m_{k, p}\right) \in \mathbb{N}^{k}$ approximate 
the semiclassical ray $\mathbb{R}_{>0} \cdot\left(r_{1}, \ldots, r_{k}\right) \in \mathbb{R}_{>0}^{k}$ with a remainder $o(p)$, as $p \rightarrow \infty$. For details see Corollary 5.11 .

We consider further the situation when the metrics $h_{p}$ on the bundles $L_{p}$ are smooth. In the case of powers $\left(L_{p}, h_{p}\right)=\left(L^{p}, h^{p}\right)$ of an ample line bundle $(L, h)$, the first order asymptotics of the Bergman kernel function was showed by Tian [T1]. A simple proof for this was given by Berndtsson in [Be, Section 2]. Adapting his methods to our situation we prove the following:

Theorem 1.3. Let $(X, \omega)$ be a compact Kähler manifold of dimension $n$. Let $\left(L_{p}, h_{p}\right), p \geq 1$, be a sequence of holomorphic line bundles on $X$ with Hermitian metrics $h_{p}$ of class $\mathscr{C}^{3}$ whose curvature forms verify (1) and such that

$$
\varepsilon_{p}:=\left\|h_{p}\right\|_{3}^{1 / 3} a_{p}^{-1 / 2} \rightarrow 0 \text { as } p \rightarrow \infty .
$$

Then there exist $C>0$ depending only on $(X, \omega)$ and $p_{0} \in \mathbb{N}$ such that

$$
\left|P_{p}(x) \frac{\omega_{x}^{n}}{c_{1}\left(L_{p}, h_{p}\right)_{x}^{n}}-1\right| \leq C \varepsilon_{p}^{2 / 3}
$$

holds for every $x \in X$ and $p>p_{0}$.

Here $\left\|h_{p}\right\|_{3}$ denotes the sup-norm of the derivatives of $h_{p}$ of order at most three with respect to a reference cover of $X$ as defined in Section 2.5. Theorem 1.3 is a generalization of the first order asymptotic expansion of the Bergman kernel [Be, Ca, DLM, MM1, MM2, [R, T1, Z] for $\left(L_{p}, h_{p}\right)=\left(L^{p}, h^{p}\right)$, where $(L, h)$ is a positive line bundle with smooth metric $h$ (see Remark 3.3).

The paper is organized as follows. In Section 2 we collect the necessary technical facts about complex spaces, plurisubharmonic functions, currents and singular Hermitian metrics on holomorphic line bundles. Section 3 is devoted to the proofs of Theorems 1.1, 1.2, 1.3, In Section 4 we give some applications of these theorems to the equidistribution of zeros of random sequences of holomorphic sections and to the approximation of certain positive closed currents on $X$ by currents of integration along zeros of holomorphic sections. In Section 5 we specialize these results to the cases when $\left(L_{p}, h_{p}\right)$ are the powers of a single line bundle, or tensor products of powers of several line bundles. We also show that some interesting cases like Satake-Baily-Borel compactifications, singular Kähler-Einstein metrics on varieties of general type or Kähler metrics with conical singularities fit into this framework.

\section{Preliminaries}

We recall here a few notions and results that will be needed throughout the paper.

2.1. Plurisubharmonic functions and currents on analytic spaces. Let $X$ be a complex space. A chart $(U, \tau, V)$ on $X$ is a triple consisting of an open set $U \subset X$, a closed complex space $V \subset G \subset \mathbb{C}^{N}$ in an open set $G$ of $\mathbb{C}^{N}$ and a biholomorphic map $\tau: U \rightarrow V$ (in the category of complex spaces). The map $\tau: U \rightarrow G \subset \mathbb{C}^{N}$ is called a local embedding of the complex space $X$. We write

$$
X=X_{\text {reg }} \cup X_{\text {sing }},
$$


where $X_{\text {reg }}$ (resp. $X_{\text {sing }}$ ) is the set of regular (resp. singular) points of $X$. Recall that a reduced complex space $(X, \mathscr{O})$ is called normal if for every $x \in X$ the local ring $\mathscr{O}_{x}$ is integrally closed in its quotient field $\mathscr{M}_{x}$. Every normal complex space is locally irreducible and locally pure dimensional, cf. [GR2, p. 125], $X_{\text {sing }}$ is a closed complex subspace of $X$ with codim $X_{\text {sing }} \geq 2$. Moreover, Riemann's second extension theorem holds on normal complex spaces [GR2, p. 143]. In particular, every holomorphic function on $X_{\text {reg }}$ extends uniquely to a holomorphic function on $X$.

Let $X$ be a complex space. A continuous (resp. smooth) function on $X$ is a function $\varphi: X \rightarrow \mathbb{C}$ such that for every $x \in X$ there exists a local embedding $\tau: U \rightarrow G \subset \mathbb{C}^{N}$ with $x \in U$ and a continuous (resp. smooth) function $\widetilde{\varphi}: G \rightarrow \mathbb{C}$ such that $\left.\varphi\right|_{U}=\widetilde{\varphi} \circ \tau$.

A (strictly) plurisubharmonic (psh) function on $X$ is a function $\varphi: X \rightarrow[-\infty, \infty)$ such that for every $x \in X$ there exists a local embedding $\tau: U \rightarrow G \subset \mathbb{C}^{N}$ with $x \in U$ and a (strictly) psh function $\widetilde{\varphi}: G \rightarrow[-\infty, \infty)$ such that $\left.\varphi\right|_{U}=\widetilde{\varphi} \circ \tau$. If $\widetilde{\varphi}$ can be chosen continuous (resp. smooth), then $\varphi$ is called a continuous (resp. smooth) psh function. The definition is independent of the chart, as is seen from [N, Lemma 4]. It is clear that a continuous psh function is continuous; by a theorem of Richberg $[R]$ the converse also holds true, i.e., a continuous function which is (strictly) psh is also continuous (strictly) psh. The analogue of Riemann's second extension theorem for psh functions holds on normal complex spaces [GR1, Satz 4]. In particular, every psh function on $X_{\text {reg }}$ extends uniquely to a psh function on $X$. We let $P S H(X)$ denote the set of psh functions on $X$, and refer to [GR1], [N], [FN], [D3] for the properties of psh functions on $X$. We recall here that psh functions on $X$ are locally integrable with respect to the area measure on $X$ given by any local embedding $\tau: U \rightarrow G \subset \mathbb{C}^{N}$ [D3, Proposition 1.8].

Let $X$ be a complex space of pure dimension $n$. We consider currents on $X$ as defined in [D3]. The sheaf of smooth $(p, q)$-forms on $X$ is defined at first locally. Let $\tau: U \rightarrow$ $G \subset \mathbb{C}^{N}$ be a local embedding. We define $\Omega^{p, q}(U)$ to be the image of the morphism $\tau^{*}: \Omega^{p, q}(G) \rightarrow \Omega^{p, q}\left(U_{\text {reg }}\right)$. It can be easily seen that there exists a sheaf $\Omega^{p, q}$ on $X$ whose space of sections on any domain $U$ of local embedding is $\Omega^{p, q}(U)$. Let $\mathcal{D}^{p, q}(X) \subset \Omega^{p, q}(X)$ be the space of forms with compact support, endowed with the inductive limit topology. The dual $\mathcal{D}_{p, q}^{\prime}(X)$ of $\mathcal{D}_{p, q}(X)$ is the space of currents of bidimension $(p, q)$, or bidegree $(n-p, n-q)$, on $X$. In particular, if $v \in P S H(X)$ then $d d^{c} v \in \mathcal{D}_{n-1, n-1}^{\prime}(X)$ is positive and closed.

Let $\mathscr{T}(X)$ be the space of positive closed currents of bidegree $(1,1)$ on $X$ which have local psh potentials: $T \in \mathscr{T}(X)$ if every $x \in X$ has a neighborhood $U$ (depending on $T$ ) such that there exists a psh function $v$ on $U$ with $T=d d^{c} v$ on $U \cap X_{\text {reg. }}$. Most of the currents considered here, like the curvature currents $c_{1}\left(L_{p}, h_{p}\right)$ and the Fubini-Study currents $\gamma_{p}$ from Theorem 1.1, belong to $\mathscr{T}(X)$. Suppose now that $Y$ is a normal analytic space, $f: Y \longrightarrow X$ is a holomorphic map, and $T \in \mathscr{T}(X)$ is such that if $v$ is a local psh potential of $T$ then $v \circ f$ is not identically $-\infty$ on an open set of $Y$. Then the pullback $f^{*} T \in \mathscr{T}(Y)$ is a well-defined current whose local psh potentials are $v \circ f$. Some interesting open questions that we will not pursue here are the following: Does every positive closed current of bidegree $(1,1)$ on $X$ belong to $\mathscr{T}(X)$ ? Is $\mathscr{T}(X)$ closed in the weak* topology on currents? If $T_{k}, T \in \mathscr{T}(X)$ and $T_{k} \rightarrow T$ weakly on $X$, does $\left\{f^{*} T_{k}\right\}$ converge to $f^{*} T$ weakly on $Y$ ? 
A Kähler form on $X$ is a current $\omega \in \mathscr{T}(X)$ whose local potentials extend to smooth strictly psh functions in local embeddings of $X$ to Euclidean spaces. We call $X$ a Kähler space if $X$ admits a Kähler form (see also [Gr, p. 346], [O], [EGZ, Sec. 5]). A Kähler form is a particular case of a Hermitian form on a complex space. Recall that a Hermitian form on a complex manifold is a smooth positive $(1,1)$-form and can be identified to a Hermitian metric. Now, a Hermitian form on a complex space $X$ is defined as a smooth $(1,1)$-form $\omega$ on $X$ such that for every point $x \in X$ there exists a local embedding $\tau$ : $U \rightarrow G \subset \mathbb{C}^{N}$ with $x \in U$ and a Hermitian form $\widetilde{\omega}$ on $G$ with $\omega=\tau^{*} \widetilde{\omega}$ on $U \cap X_{\text {reg }}$. A Hermitian form on a paracompact complex space $X$ is constructed as usual by a partition of unity argument. A Hermitian form $\omega$ on $X$ clearly induces a Hermitian form in the usual sense (and thus a Hermitian metric) on $X_{\text {reg }}$. Note that $\omega^{n} / n$ ! gives locally an area measure on $X$.

2.2. Singular Hermitian holomorphic line bundles on analytic spaces. Let $L$ be a holomorphic line bundle on a normal Kähler space $(X, \omega)$. The notion of singular Hermitian metric $h$ on $L$ is defined exactly as in the smooth case (see [D4], [MM1, p. 97])): if $e_{\alpha}$ is a holomorphic frame of $L$ over an open set $U_{\alpha} \subset X$ then $\left|e_{\alpha}\right|_{h}^{2}=e^{-2 \varphi_{\alpha}}$ where $\varphi_{\alpha} \in L_{l o c}^{1}\left(U_{\alpha}, \omega^{n}\right)$. If $g_{\alpha \beta}=e_{\beta} / e_{\alpha} \in \mathcal{O}_{X}^{*}\left(U_{\alpha} \cap U_{\beta}\right)$ are the transition functions of $L$ then $\varphi_{\alpha}=\varphi_{\beta}+\log \left|g_{\alpha \beta}\right|$. The curvature current $c_{1}(L, h) \in \mathcal{D}_{n-1, n-1}^{\prime}(X)$ of $h$ is defined by $c_{1}(L, h)=d d^{c} \varphi_{\alpha}$ on $U_{\alpha} \cap X_{\text {reg. }}$. We will denote by $h^{p}$ the singular Hermitian metric induced by $h$ on $L^{p}:=L^{\otimes p}$. If $c_{1}(L, h) \geq 0$ then the weight $\varphi_{\alpha}$ is psh on $U_{\alpha} \cap X_{\text {reg }}$ and since $X$ is normal it extends to a psh function on $U_{\alpha}$ [GR1, Satz 4], hence $c_{1}(L, h) \in \mathscr{T}(X)$.

Let $L$ be a holomorphic line bundle on a compact normal Kähler space $(X, \omega)$. Then the space $H^{0}(X, L)$ of holomorphic sections of $L$ is finite dimensional. This is a special case of the Cartan-Serre finiteness theorem; an elementary proof using the Schwarz lemma can be found in [A, Théorème 1, p.27]. The space $H_{(2)}^{0}(X, L)$ defined as in (3) is therefore also finite dimensional.

If $P_{p}$ and $\gamma_{p}$ are the Bergman kernel functions, resp. the Fubini-Study currents, of the spaces $H_{(2)}^{0}\left(X, L_{p}\right)$ from Theorem 1.1, it follows from (4) that $\log P_{p} \in L^{1}\left(X, \omega^{n}\right)$ and

$$
\gamma_{p}-c_{1}\left(L_{p}, h_{p}\right)=\frac{1}{2} d d^{c} \log P_{p} .
$$

Moreover, as in [CM1, CM2], one has the following variational formula,

$$
P_{p}(x)=\max \left\{|S(x)|_{h_{p}}^{2}: S \in H_{(2)}^{0}\left(X, L_{p}\right),\|S\|_{p}=1\right\} .
$$

This is valid for all $x \in X$ such that $\varphi_{p}(x)>-\infty$, where $\varphi_{p}$ is a local weight of the metric $h_{p}$ near $x$.

2.3. Resolution of singularities. Bierstone and Milman constructed a resolution of singularities of a compact analytic space $X, \pi: \widetilde{X} \longrightarrow X$, by a finite sequence of blow-ups with smooth center $\sigma_{j}: X_{j+1} \longrightarrow X_{j}, X_{0}=X$, with the property that for any local embed$\left.\operatorname{ding} X\right|_{U} \hookrightarrow \mathbb{C}^{N}$ this sequence of blow-ups is induced by the embedded desingularization of $\left.X\right|_{U}[\overline{\mathrm{BM}}$, Theorem 13.2]. In [GM, sec. 6] it is shown that the embedded desingularization of $\left.X\right|_{U} \hookrightarrow \mathbb{C}^{N}$ by a finite sequence of blow-ups with smooth center is equivalent to a single blow-up along a coherent sheaf of ideals $\mathcal{I}$ whose support is $\left.X_{\text {sing }}\right|_{U}$. It follows 
that every point $x \in X$ has a neighborhood $U \subset X$ for which there exists an ideal $\mathcal{I}_{U}$ generated by finitely many holomorphic functions on $U$ such that $\pi: \pi^{-1}(U) \longrightarrow U$ is equivalent to the blow-up of $\left.X\right|_{U}$ along $\mathcal{I}_{U}$.

We fix throughout the paper a resolution of singularities $\pi: \widetilde{X} \longrightarrow X$ of our compact normal space $X$ as described above, and we denote by $\Sigma=\pi^{-1}\left(X_{\text {sing }}\right)$ the exceptional divisor. Note that $\pi: \widetilde{X} \backslash \Sigma \longrightarrow X_{\text {reg }}$ is a biholomorphism. We will need to consider the singular Hermitian holomorphic line bundles $\left(\pi^{*} L_{p}, \pi^{*} h_{p}\right)$ obtained by pulling back $\left(L_{p}, h_{p}\right)$ to $\widetilde{X}$ by the map $\pi$, and their spaces of $L^{2}$-holomorphic sections

$$
H_{(2)}^{0}\left(\widetilde{X}, \pi^{*} L_{p}\right)=\left\{\widetilde{S} \in H^{0}\left(\widetilde{X}, \pi^{*} L_{p}\right): \int_{\widetilde{X}}|\widetilde{S}|_{\pi^{*} h_{p}}^{2} \frac{\pi^{*} \omega^{n}}{n !}<\infty\right\} .
$$

Lemma 2.1. The map $\pi^{*}: H_{(2)}^{0}\left(X, L_{p}\right) \longrightarrow H_{(2)}^{0}\left(\widetilde{X}, \pi^{*} L_{p}\right)$ is an isometry and the Bergman kernel function of $H_{(2)}^{0}\left(\widetilde{X}, \pi^{*} L_{p}\right)$ is $\widetilde{P}_{p}=P_{p} \circ \pi$.

Proof. Let $S_{1}^{p}, \ldots, S_{d_{p}}^{p}$ be an orthonormal basis of $H_{(2)}^{0}\left(X, L_{p}\right)$ and $\widetilde{S}_{j}^{p}=\pi^{*} S_{j}^{p}$ be the induced sections of $\pi^{*} L^{p}$. Then $\left|\widetilde{S}_{j}^{p}\right|_{\pi^{*} h_{p}}=\left|S_{j}^{p}\right|_{h_{p}} \circ \pi$ and

$$
\int_{\widetilde{X}}\left|\widetilde{S}_{j}^{p}\right|_{\pi^{*} h_{p}}^{2} \frac{\pi^{*} \omega^{n}}{n !}=\int_{\widetilde{X} \backslash \Sigma}\left|\widetilde{S}_{j}^{p}\right|_{\pi^{*} h_{p}}^{2} \frac{\pi^{*} \omega^{n}}{n !}=\int_{X_{\text {reg }}}\left|S_{j}^{p}\right|_{h_{p}}^{2} \frac{\omega^{n}}{n !}=1 .
$$

Suppose now that $\widetilde{S} \in H_{(2)}^{0}\left(\widetilde{X}, \pi^{*} L_{p}\right)$ is orthogonal to all $\widetilde{S}_{j}^{p}$ and let $S$ be the induced

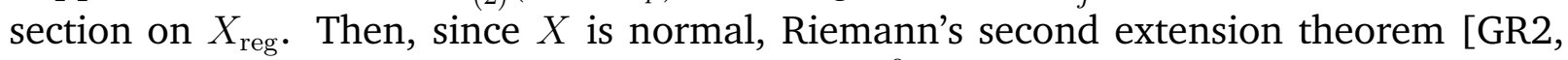
p. 143] shows that $S$ extends to a section $S \in H^{0}\left(X, L_{p}\right)$. By the preceding argument, $S \in H_{(2)}^{0}\left(X, L_{p}\right)$ is orthogonal to all $S_{j}^{p}$, hence $S=0$ and $\widetilde{S}=0$. It follows that $\left\{\widetilde{S}_{j}^{p}\right\}$ is an orthonormal basis of $H_{(2)}^{0}\left(\widetilde{X}, \pi^{*} L_{p}\right)$ and

$$
\widetilde{P}_{p}=\sum_{j=1}^{d_{p}}\left|\widetilde{S}_{j}^{p}\right|_{\pi^{*} h_{p}}^{2}=\sum_{j=1}^{d_{p}}\left|S_{j}^{p}\right|_{h_{p}}^{2} \circ \pi=P_{p} \circ \pi .
$$

Adapting the proof of [Moi, Lemma 1] to our situation we obtain the following lemma, whose proof is included for the convenience of the reader.

Lemma 2.2. Let $(X, \omega)$ be a compact (reduced) Hermitian space and $\pi: \widetilde{X} \longrightarrow X$ be a resolution of singularities as described above, with exceptional divisor $\Sigma$. Then there exists a smooth Hermitian metric $\theta$ on $F=\mathcal{O}_{\tilde{X}}(-\Sigma)$ and $C>0$ such that $\Omega=C \pi^{*} \omega+c_{1}(F, \theta)$ is a Hermitian form on $\tilde{X}$ and $\Omega \geq \pi^{*} \omega$. If $\omega$ is Kähler, then $\Omega$ is Kähler, too.

Proof. As in the proof of [Moi, Lemma 1], we can find an open cover $X=\bigcup_{k=1}^{N} U_{k}$ with the following properties:

(i) There exist a local embedding $\tau_{k}: U_{k} \rightarrow B_{k}$ into a ball $B_{k} \subset \mathbb{C}^{l_{k}}$ and a smooth Hermitian form $\omega_{k}$ on $B_{k}$ such that $\omega=\tau_{k}^{*} \omega_{k}$ on $U_{k} \cap X_{\text {reg. }}$. Hence we may assume that $U_{k} \subset B_{k}$ and $\omega$ is the restriction of $\omega_{k}$ to $U_{k} \cap X_{\text {reg }}$. Choose a strictly psh function $\eta_{k}$ on 
$B_{k}$ such that $\omega_{k} \geq d d^{c} \eta_{k}$ on $B_{k}$. If $\omega$ is Kähler, we choose $\eta_{k}$ such that $\omega_{k}=d d^{c} \eta_{k}$ on $B_{k}$, hence $\omega=d d^{c} \eta_{k}$ on $U_{k} \cap X_{\text {reg }}$.

(ii) There exist finitely many holomorphic functions $f_{0 k}, \ldots, f_{N_{k} k} \in \mathcal{O}_{X}\left(U_{k}\right)$ such that over $U_{k}$ the map $\pi: \pi^{-1}\left(U_{k}\right) \longrightarrow U_{k}$ and the line bundle $\left.\mathcal{O}_{\tilde{X}}(\Sigma)\right|_{\pi^{-1}\left(U_{k}\right)}$ are described as follows. If $\Gamma_{k} \subset U_{k} \times \mathbb{P}^{N_{k}}$ is the graph of the meromorphic map $x \mapsto\left[f_{0 k}(x): \ldots: f_{N_{k} k}(x)\right]$ with canonical projections $\pi_{1 k}: \Gamma_{k} \longrightarrow U_{k}$ and $\pi_{2 k}: \Gamma_{k} \longrightarrow \mathbb{P}^{N_{k}}$ then $\pi: \pi^{-1}\left(U_{k}\right) \longrightarrow U_{k}$ coincides with $\pi_{1 k}: \Gamma_{k} \longrightarrow U_{k}$ and $\left.\mathcal{O}_{\tilde{X}}(\Sigma)\right|_{\pi^{-1}\left(U_{k}\right)}$ can be identified with the line bundle $\pi_{2 k}^{*} \mathcal{O}_{\mathbb{P}^{N_{k}}}(-1)$ on $\Gamma_{k}$.

Let $\left[y_{0 k}: \ldots: y_{N_{k} k}\right]$ denote the homogeneous coordinates on $\mathbb{P}^{N_{k}}, G_{i k}=\left\{y_{i k} \neq 0\right\} \subset$ $\mathbb{P}^{N_{k}}, \widetilde{G}_{i k}=\pi_{2 k}^{-1}\left(G_{i k}\right) \subset \Gamma_{k}=\pi^{-1}\left(U_{k}\right)$. The transition functions of $\mathcal{O}_{\mathbb{P}^{N_{k}}}(-1)$ are $y_{i k} / y_{j k}$ and we endow $\mathcal{O}_{\mathbb{P}^{N_{k}}}(-1)$ with a smooth Hermitian metric with weights $\psi_{i k}^{\prime}$ on $G_{i k}$ such that $-\psi_{i k}^{\prime}$ is strictly psh. The corresponding transition functions of $\left.\mathcal{O}_{\tilde{X}}(\Sigma)\right|_{\pi^{-1}\left(U_{k}\right)}$ are $\widetilde{g}_{i j, k}=\left(f_{i k} \circ \pi_{1 k}\right) /\left(f_{j k} \circ \pi_{1 k}\right)$ on $\widetilde{G}_{i k} \cap \widetilde{G}_{j k}$, and we denote by $\psi_{i k}$ the weights of the induced Hermitian metric. Then $-\psi_{i k}$ is a smooth psh function on $\widetilde{G}_{i k}$ and $\psi_{i k}=\psi_{j k}+\log \left|\widetilde{g}_{i j, k}\right|$ on $\widetilde{G}_{i k} \cap \widetilde{G}_{j k}$.

Consider the open cover $\left\{\widetilde{G}_{i k}: 1 \leq k \leq N, 0 \leq i \leq N_{k}\right\}$ of $\widetilde{X}$. Note that $\pi^{-1}\left(U_{k}\right)=$ $\bigcup_{i=0}^{N_{k}} \widetilde{G}_{i k}$ and that $\mathcal{O}_{\widetilde{X}}(\Sigma)$ is trivial on $\widetilde{G}_{i k}$. We denote the transition functions of $\mathcal{O}_{\widetilde{X}}(\Sigma)$ by $g_{i_{1} k_{1}, i_{2} k_{2}} \in \mathcal{O}\left(\widetilde{G}_{i_{1} k_{1}} \cap \widetilde{G}_{i_{2} k_{2}}\right)$. Hence

$$
\psi_{i_{1} k}=\psi_{i_{2} k}+\log \left|g_{i_{1} k, i_{2} k}\right|, \quad g_{i_{1} k, i_{2} k}=\widetilde{g}_{i_{1} i_{2}, k} .
$$

We now construct a smooth Hermitian metric on $\mathcal{O}_{\widetilde{X}}(\Sigma)$ with weights $s_{i k}$ on $\widetilde{G}_{i k}$. Let $\left\{\rho_{k}\right\}_{1 \leq k \leq N}$ be a smooth partition of unity on $X$ so that $\operatorname{supp} \rho_{k} \subset U_{k}$. For a fixed $k_{1} \in$ $\{1, \ldots, N\}$ let $\varphi_{k_{1}}^{i k}$ be a function defined on $\pi^{-1}\left(U_{k_{1}}\right) \cap \widetilde{G}_{i k}$ as follows: if $x \in \widetilde{G}_{i_{1} k_{1}}$ then

$$
\varphi_{k_{1}}^{i k}(x)=\psi_{i_{1} k_{1}}(x)+\log \left|g_{i k, i_{1} k_{1}}(x)\right| .
$$

Note that $\varphi_{k_{1}}^{i k}$ is well defined and smooth on $\pi^{-1}\left(U_{k_{1}}\right) \cap \widetilde{G}_{i k}$. Indeed, if $x \in \widetilde{G}_{i_{1} k_{1}} \cap \widetilde{G}_{i_{1}^{\prime} k_{1}} \cap \widetilde{G}_{i k}$ then $g_{i_{1}^{\prime} k_{1}, i_{1} k_{1}} \cdot g_{i_{1} k_{1}, i k} \cdot g_{i k, i_{1}^{\prime} k_{1}}=1$ so

$$
\begin{aligned}
\psi_{i_{1}^{\prime} k_{1}}(x)+\log \left|g_{i k, i_{1}^{\prime} k_{1}}(x)\right| & =\psi_{i_{1} k_{1}}(x)+\log \left|g_{i_{1}^{\prime} k_{1}, i_{1} k_{1}}(x)\right|+\log \left|g_{i k, i_{1}^{\prime} k_{1}}(x)\right| \\
& =\psi_{i_{1} k_{1}}(x)+\log \left|g_{i k, i_{1} k_{1}}(x)\right| .
\end{aligned}
$$

Next we define

$$
s_{i k}=\sum_{k_{1}=1}^{N}\left(\rho_{k_{1}} \circ \pi\right) \varphi_{k_{1}}^{i k} \text { on } \widetilde{G}_{i k} .
$$

We claim that $s_{i k}=s_{i^{\prime} k^{\prime}}+\log \left|g_{i k, i^{\prime} k^{\prime}}\right|$ on $\widetilde{G}_{i k} \cap \widetilde{G}_{i^{\prime} k^{\prime}}$, so $\left\{s_{i k}\right\}$ defines a smooth Hermitian metric on $\mathcal{O}_{\widetilde{X}}(\Sigma)$. For this we show that if $k_{1} \in\{1, \ldots, N\}$ then

$$
\left(\rho_{k_{1}} \circ \pi\right) \varphi_{k_{1}}^{i k}=\left(\rho_{k_{1}} \circ \pi\right)\left(\varphi_{k_{1}}^{i^{\prime} k^{\prime}}+\log \left|g_{i k, i^{\prime} k^{\prime}}\right|\right)
$$


Let $x \in \pi^{-1}\left(U_{k_{1}}\right)$ and assume $x \in \widetilde{G}_{i_{1} k_{1}} \cap \widetilde{G}_{i k} \cap \widetilde{G}_{i^{\prime} k^{\prime}}$. Since $g_{i^{\prime} k^{\prime}, i_{1} k_{1}} \cdot g_{i_{1} k_{1}, i k} \cdot g_{i k, i^{\prime} k^{\prime}}=1$ we obtain

$$
\begin{aligned}
\varphi_{k_{1}}^{i k}(x) & =\psi_{i_{1} k_{1}}(x)+\log \left|g_{i k, i_{1} k_{1}}(x)\right|=\psi_{i_{1} k_{1}}(x)+\log \left|g_{i^{\prime} k^{\prime}, i_{1} k_{1}}(x)\right|+\log \left|g_{i k, i^{\prime} k^{\prime}}(x)\right| \\
& =\varphi_{k_{1}}^{i^{\prime} k^{\prime}}(x)+\log \left|g_{i k, i^{\prime} k^{\prime}}(x)\right|
\end{aligned}
$$

which proves our claim.

We finally show that the desired metric $\theta$ on $F$ is the metric defined by the weights $\left\{-s_{i k}\right\}$. By a standard compactness argument it suffices to prove that for every $x \in \widetilde{G}_{i k}$ there exists $C_{x}>0$ such that for $C>C_{x}$ the function $C \eta_{k} \circ \pi-s_{i k}$ is strictly psh at $x$. We write $T_{x} \widetilde{X}=E_{x} \oplus F_{x}$, where $E_{x}=\operatorname{ker} d \pi(x)$. Note that the Levi form (see e.g. [H2, p. 228] for the definition) of the function $\eta_{k} \circ \pi$ is given by

$$
\mathcal{L}\left(\eta_{k} \circ \pi\right)(x)\left(t, t^{\prime}\right)=\mathcal{L} \eta_{k}(\pi(x))\left(d \pi(x)(t), d \pi(x)\left(t^{\prime}\right)\right),
$$

SO

$$
\mathcal{L}\left(\eta_{k} \circ \pi\right)(x)\left(t, t^{\prime}\right)=0, \forall t \in T_{x} \tilde{X}, t^{\prime} \in E_{x} .
$$

Moreover, since $\eta_{k}$ is strictly psh at $\pi(x) \in B_{k}$ and $d \pi(x)$ is injective on $F_{x}$ we deduce that

$$
\mathcal{L}\left(\eta_{k} \circ \pi\right)(x)(t, t)>0, \forall t \in F_{x} \backslash\{0\} .
$$

The formula of $s_{i k}$ implies that for each $t \in E_{x}$

$$
-\mathcal{L} s_{i k}(x)(t, t)=-\sum_{k_{1}=1}^{N} \rho_{k_{1}}(\pi(x)) \mathcal{L} \psi_{i_{1} k_{1}}(x)(t, t) \geq 0,
$$

since each function $-\psi_{i_{1} k_{1}}$ is psh on $\widetilde{G}_{i_{1} k_{1}}$. If $\rho_{k_{1}}(\pi(x))>0$ we may assume that $x \in$ $\widetilde{G}_{i_{1} k_{1}} \cap \widetilde{G}_{i k}$ and we will show that $-\mathcal{L} \psi_{i_{1} k_{1}}(x)(t, t)>0$ for all $t \in E_{x} \backslash\{0\}$. As $\pi^{-1}\left(U_{k_{1}}\right)=$ $\Gamma_{k_{1}} \subset U_{k_{1}} \times \mathbb{P}^{N_{k_{1}}} \subset B_{k_{1}} \times \mathbb{P}^{N_{k_{1}}}, x$ has a neighborhood $\widetilde{U} \subset \widetilde{X}$ such that $\widetilde{U} \subset B_{k_{1}} \times G_{i_{1} k_{1}}$. Recall that on $\widetilde{U}, \psi_{i_{1} k_{1}}=\psi_{i_{1} k_{1}}^{\prime} \circ \pi_{2 k_{1}}$ and $\pi=\pi_{1 k_{1}}$. We consider $\pi_{1 k_{1}}, \pi_{2 k_{1}}$ as restrictions of the canonical projections $\pi_{1 k_{1}}: B_{k_{1}} \times G_{i_{1} k_{1}} \longrightarrow B_{k_{1}}, \pi_{2 k_{1}}: B_{k_{1}} \times G_{i_{1} k_{1}} \longrightarrow G_{i_{1} k_{1}}$. Since $t \in E_{x} \subset \operatorname{ker} d \pi_{1 k_{1}}(x)$ and $t \neq 0$ it follows that $d \pi_{2 k_{1}}(x)(t) \neq 0$. Therefore

$$
-\mathcal{L} \psi_{i_{1} k_{1}}(x)(t, t)=-\mathcal{L} \psi_{i_{1} k_{1}}^{\prime}\left(\pi_{2 k_{1}}(x)\right)\left(d \pi_{2 k_{1}}(x)(t), d \pi_{2 k_{1}}(x)(t)\right)>0,
$$

as $-\psi_{i_{1} k_{1}}^{\prime}$ is strictly psh on $G_{i_{1} k_{1}}$. This yields

$$
-\mathcal{L} s_{i k}(x)(t, t)>0, \forall t \in E_{x} \backslash\{0\} .
$$

By (8), (9) and (10) we conclude that there exists $C_{x}>0$ such that if $C>C_{x}$ then $\mathcal{L}\left(C \eta_{k} \circ \pi-s_{i k}\right)(x)(t, t)>0$ for all $t \in T_{x} \widetilde{X} \backslash\{0\}$. This finishes the proof.

We look now at the nature of the base space $X$ as implied by the hypotheses made on the curvature of the bundles involved in our results. Recall that a compact irreducible complex space $X$ of dimension $n$ is called Moishezon if $X$ possesses $n$ algebraically independent meromorphic functions, i.e., if the transcendence degree of the field of meromorphic functions on $X$ equals the complex dimension of $X$. Let $X^{\prime}$ and $X$ be compact irreducible spaces and $h: X^{\prime} \rightarrow X$ be a proper modification. Then $h$ induces an isomorphism of the fields of meromorphic functions on $X^{\prime}$ and $X$, respectively, 
[MM1, Theorem 2.1.18], hence $X^{\prime}$ is Moishezon if and only if $X$ is Moishezon. Moishezon [Moi] showed that if $X$ is a Moishezon space, then there exists a proper modification $h: X^{\prime} \rightarrow X$, obtained by a finite number of blow-ups with smooth centers, such that $X^{\prime}$ is a projective algebraic manifold (for a proof see also [MM1, Theorem 2.2.16]).

Lemma 2.2 yields in particular the following:

Proposition 2.3. If $(X, \omega)$ is a compact (reduced) Hermitian space endowed with a singular Hermitian holomorphic line bundle $(L, h)$ such that $c_{1}(L, h) \geq \varepsilon \omega$ for some constant $\varepsilon>0$ then $X$ is Moishezon.

Proof. Let $\pi: \widetilde{X} \longrightarrow X$ be a resolution of singularities as in Lemma 2.2, with exceptional divisor $\Sigma$ and with Hermitian form $\Omega=C \pi^{*} \omega+c_{1}(F, \theta)$, where $F=\mathcal{O}_{\tilde{X}}(-\Sigma)$. Consider the line bundles $E_{p}=\pi^{*} L^{p} \otimes F$ with singular Hermitian metrics $\theta_{p}=\pi^{*} h^{p} \otimes \theta$. Then

$$
c_{1}\left(E_{p}, \theta_{p}\right)=p \pi^{*} c_{1}(L, h)+c_{1}(F, \theta) \geq p \varepsilon \pi^{*} \omega+c_{1}(F, \theta) \geq \Omega,
$$

provided that $p \varepsilon \geq C$. Hence $\widetilde{X}$ carries a singular Hermitian holomorphic line bundle with strictly positive curvature in the sense of currents. By [JS] (see also [MM1, Theorem 2.3.8]) it follows that $\widetilde{X}$ is Moishezon and hence $X$, too. If $(X, \omega)$ is Kähler, then $\widetilde{X}$ is already projective. Indeed, $(\widetilde{X}, \Omega)$ is Kähler and Moishezon, so by a theorem of Moishezon is projective (see e. g. [MM1, Theorem 2.2.26]).

The paper $[\overline{\mathrm{M}}]$ (see also [MM1, Theorems 3.4.10 and 3.4.14 ]) gives an integral criterion for a complex space with isolated singularities to be Moishezon, generalizing the criterion of Siu and Demailly from the smooth case.

We recall next the following projectivity criterion.

Proposition 2.4 (Grauert). If $(X, \omega)$ is a compact (reduced) Hermitian space endowed with a $\mathscr{C}^{2}$ Hermitian holomorphic line bundle $(L, h)$ such that $c_{1}(L, h) \geq \varepsilon \omega$ for some constant $\varepsilon>0$, then $L$ is ample and $X$ is projective.

This follows from [Gr, Satz 2, p.343], see also [Gr, Satz 3, p.346]. If $X$ is normal, Grauert actually shows more: if $\Omega$ is a continuous Kähler metric whose de Rham cohomology class is integral, then there exists a Hermitian holomorphic line bundle $(L, h)$ with $\mathscr{C}^{2}$ Hermitian metric and $c_{1}(L, h)=\Omega$; hence $L$ is ample and $X$ is projective.

2.4. $L^{2}$-estimates for $\bar{\partial}$. The following version of Demailly's estimates for the $\bar{\partial}$ operator [D2, Théorème 5.1] will be needed in our proofs.

Theorem 2.5 ([D2] ). Let $Y, \operatorname{dim} Y=n$, be a complete Kähler manifold and let $\Omega$ be a Kähler form on $Y$ (not necessarily complete) such that its Ricci form $\operatorname{Ric}_{\Omega} \geq-2 \pi B \Omega$ on $Y$, for some constant $B>0$. Let $\left(L_{p}, h_{p}\right)$ be singular Hermitian holomorphic line bundles on $Y$ such that $c_{1}\left(L_{p}, h_{p}\right) \geq 2 a_{p} \Omega$, where $a_{p} \rightarrow \infty$ as $p \rightarrow \infty$, and fix $p_{0}$ such that $a_{p} \geq B$ for all $p>p_{0}$. If $p>p_{0}$ and $g \in L_{0,1}^{2}\left(Y, L_{p}, l o c\right)$ verifies $\bar{\partial} g=0$ and $\int_{Y}|g|_{h_{p}}^{2} \Omega^{n}<\infty$ then there exists $u \in L_{0,0}^{2}\left(Y, L_{p}, l o c\right)$ such that $\bar{\partial} u=g$ and $\int_{Y}|u|_{h_{p}}^{2} \Omega^{n} \leq \frac{1}{a_{p}} \int_{Y}|g|_{h_{p}}^{2} \Omega^{n}$.

Proof. We write $L_{p}=F_{p} \otimes K_{Y}$, where $F_{p}=L_{p} \otimes K_{Y}^{-1}$, and the canonical line bundle $K_{Y}$ is endowed with the metric $h^{K_{Y}}$ induced by $\Omega$. If $\theta_{p}=h_{p} \otimes h^{K_{Y}^{-1}}$ is the metric induced on 
$F_{p}$ then

$$
c_{1}\left(F_{p}, \theta_{p}\right)=c_{1}\left(L_{p}, h_{p}\right)-c_{1}\left(K_{Y}, h^{K_{Y}}\right)=c_{1}\left(L_{p}, h_{p}\right)+\frac{1}{2 \pi} \operatorname{Ric}_{\Omega} \geq\left(2 a_{p}-B\right) \Omega \geq a_{p} \Omega
$$

for $p>p_{0}$. The theorem follows by using the isometries $L_{0, j}^{2}\left(Y, L_{p}, l o c\right) \cong L_{n, j}^{2}\left(Y, F_{p}, l o c\right)$, $j=0,1$, and applying [D2, Théorème 5.1] (see also [CM2, Corollaries 4.2 and 4.3]).

2.5. Special weights of Hermitian metrics on reference covers. Let $(X, \omega)$ be a compact Kähler manifold of dimension $n$. Let $(U, z), z=\left(z_{1}, \ldots, z_{n}\right)$, be local coordinates centered at a point $x \in X$. For $r>0$ and $y \in U$ we denote by

$$
\Delta^{n}(y, r)=\left\{z \in U:\left|z_{j}-y_{j}\right| \leq r, j=1, \ldots, n\right\}
$$

the (closed) polydisk of polyradius $(r, \ldots, r)$ centered at $y$. The coordinates $(U, z)$ are called Kähler at $y \in U$ if

$$
\omega_{z}=\frac{i}{2} \sum_{j=1}^{n} d z_{j} \wedge d \bar{z}_{j}+O\left(|z-y|^{2}\right) \text { on } U .
$$

Definition 2.6. A reference cover of $X$ consists of the following data: for $j=1, \ldots, N$, a set of points $x_{j} \in X$ and

(1) Stein open simply connected coordinate neighborhoods $\left(U_{j}, w^{(j)}\right)$ centered at $x_{j} \equiv 0$,

(2) $R_{j}>0$ such that $\Delta^{n}\left(x_{j}, 2 R_{j}\right) \Subset U_{j}$ and for every $y \in \Delta^{n}\left(x_{j}, 2 R_{j}\right)$ there exist coordinates on $U_{j}$ which are Kähler at $y$,

(3) $X=\bigcup_{j=1}^{N} \Delta^{n}\left(x_{j}, R_{j}\right)$.

Given the reference cover as above we set $R=\min R_{j}$.

We can construct a reference cover as follows: For $x \in X$ fix a Stein open simply connected neighborhood $U$ of $x \equiv 0 \in \mathbb{C}^{n}$ and fix $R>0$ such that the polydisk $\Delta^{n}(x, 2 R) \Subset U$ and for every $y \in \Delta^{n}(x, 2 R)$ there exist coordinates $(U, z)$ which are Kähler at $y$. By compactness there exist $x_{1}, \ldots, x_{N} \in X$ such that the above conditions are fulfilled.

On $U_{j}$ we consider the partial derivatives $D_{w}^{\alpha}$ of order $|\alpha|, \alpha \in \mathbb{N}^{2 n}$, corresponding to the real coordinates associated to $w=w^{(j)}$. For a function $\varphi \in \mathscr{C}^{k}\left(U_{j}\right)$ we set

$$
\|\varphi\|_{k}=\|\varphi\|_{k, w}=\sup \left\{\left|D_{w}^{\alpha} \varphi(w)\right|: w \in \Delta^{n}\left(x_{j}, 2 R_{j}\right),|\alpha| \leq k\right\} .
$$

Let $(L, h)$ be a Hermitian holomorphic line bundle on $X$, where the metric $h$ is of class $\mathscr{C}^{\ell}$. Note that $\left.L\right|_{U_{j}}$ is trivial. For $k \leq \ell$ set

$$
\begin{aligned}
\|h\|_{k, U_{j}} & =\inf \left\{\left\|\varphi_{j}\right\|_{k}: \varphi_{j} \in \mathscr{C}^{\ell}\left(U_{j}\right) \text { is a weight of } h \text { on } U_{j}\right\}, \\
\|h\|_{k} & =\max \left\{1,\|h\|_{k, U_{j}}: 1 \leq j \leq N\right\} .
\end{aligned}
$$

Recall that $\varphi_{j}$ is a weight of $h$ on $U_{j}$ if there exists a holomorphic frame $e_{j}$ of $L$ on $U_{j}$ such that $\left|e_{j}\right|_{h}=e^{-\varphi_{j}}$.

Lemma 2.7. There exists $C>1$ (depending on the reference cover) with the following property: Given any Hermitian line bundle $(L, h)$ on $X$, where $h$ is of class $\mathscr{C}^{3}$, any $j \in$ 
$\{1, \ldots, N\}$ and any $x \in \Delta^{n}\left(x_{j}, R_{j}\right)$ there exist coordinates $z=\left(z_{1}, \ldots, z_{n}\right)$ on $\Delta^{n}(x, R)$ which are centered at $x \equiv 0$ and Kähler coordinates for $x$ such that

(i) $d m \leq\left(1+C r^{2}\right) \omega^{n} / n$ ! and $\omega^{n} / n ! \leq\left(1+C r^{2}\right) d m$ hold on $\Delta^{n}(x, r)$ for any $r<R$ where $d m=d m(z)$ is the Euclidean volume relative to the coordinates $z$,

(ii) $(L, h)$ has a weight $\varphi$ on $\Delta^{n}(x, R)$ with $\varphi(z)=\sum_{j=1}^{n} \lambda_{j}\left|z_{j}\right|^{2}+\widetilde{\varphi}(z)$, where $\lambda_{j} \in \mathbb{R}$ and $|\widetilde{\varphi}(z)| \leq C\|h\|_{3}|z|^{3}$ for $z \in \Delta^{n}(x, R)$.

Proof. By the properties of a reference cover there exist coordinates $z$ on $U_{j}$ which are Kähler for $x \in \Delta^{n}\left(x_{j}, R_{j}\right)$ so $\omega=\frac{i}{2} \sum_{l=1}^{n} d z_{l} \wedge d \bar{z}_{l}+O\left(|z-x|^{2}\right)$ and $(i)$ holds with a constant $C_{j}$ uniform for $x \in \Delta^{n}\left(x_{j}, R_{j}\right)$. Let $e_{j}$ be a frame of $L$ on $U_{j}$ and $\varphi^{\prime}$ be a weight of $h$ on $U_{j}$ with $\left|e_{j}\right|_{h}=e^{-\varphi^{\prime}}$ and $\left\|\varphi^{\prime}\right\|_{3, w} \leq 2\|h\|_{3}$, cf. (12)-(13). By translation we may assume $x=0$ and write $\varphi^{\prime}(z)=\operatorname{Re} f(z)+\varphi_{2}^{\prime}(z)+\varphi_{3}^{\prime}(z)$, where $f(z)$ is a holomorphic polynomial of degree $\leq 2$ in $z, \varphi_{2}^{\prime}(z)=\sum_{k, l=1}^{n} \mu_{k l} z_{k} \bar{z}_{l}$, and $\operatorname{Re} f(z)+\varphi_{2}^{\prime}(z)$ is the Taylor polynomial of order 2 of $\varphi^{\prime}$ at 0 . Note that $\left\|\varphi^{\prime}\right\|_{3, z} \leq C_{j}^{\prime}\left\|\varphi^{\prime}\right\|_{3, w} \leq 2 C_{j}^{\prime}\|h\|_{3}$, where $\left\|\varphi^{\prime}\right\|_{3, z}$ is the sup norm on $\Delta^{n}(x, R)$ of the derivatives of order 3 of $\varphi^{\prime}$ in the coordinates $z$ and $C_{j}^{\prime}$ is a constant uniform for $x \in \Delta^{n}\left(x_{j}, R_{j}\right)$. This follows from the fact that $z, w$ are coordinates on $U_{j} \ni \Delta^{n}\left(x_{j}, 2 R_{j}\right)$. We conclude that $\left|\varphi_{3}^{\prime}(z)\right| \leq 2 C_{j}^{\prime}\|h\|_{3}|z|^{3}$ for $z \in \Delta^{n}(x, R)$.

Consider the frame $\widetilde{e}_{j}=e^{f} e_{j}$ of $L$ on $U_{j}$. Then $\left|\widetilde{e}_{j}\right|_{h}=e^{\operatorname{Re} f-\varphi^{\prime}}=e^{-\varphi}$, so $\varphi(z):=\varphi_{2}^{\prime}(z)+$ $\varphi_{3}^{\prime}(z)$ is a weight of $h$ on $U_{j}$. By a unitary change of coordinates we may assume that $\varphi(z)=\sum_{l=1}^{n} \lambda_{l}\left|z_{l}\right|^{2}+\widetilde{\varphi}(z)$. In these new coordinates $\omega^{n} / n$ ! and $\widetilde{\varphi}(z)$ satisfy the desired estimates with a constant $C_{j}$ uniform for $x \in \Delta^{n}\left(x_{j}, R_{j}\right)$. Finally we let $C=\max _{1 \leq j \leq N} C_{j}$.

\section{Proofs of Theorems 1.1, 1.2 and 1.3}

We use here the notations introduced in Section 2 and we start with two lemmas that will be needed in the proof of Theorem 1.1;

Lemma 3.1. Let $D$ be a divisor in a complex manifold $Y$ and $\theta$ be a smooth Hermitian metric on $\mathcal{O}(-D)$ over $Y$ with weight $\varphi$ over $Y \backslash D$. Then $\lim _{y \rightarrow x, y \in Y \backslash D} \varphi(y)=-\infty$ for every $x \in D$.

Proof. Let $U_{\alpha}$ be a neighborhood of $x$ where $D$ has defining function $f_{\alpha} \in \mathcal{O}\left(U_{\alpha}\right)$ and $\theta$ has weight $\varphi_{\alpha} \in \mathscr{C}^{\infty}\left(U_{\alpha}\right)$. The transition function of $\mathcal{O}(-D)$ on $U_{\alpha} \cap(Y \backslash D)$ is $g=1 / f_{\alpha}$, so $\varphi_{\alpha}=\varphi+\log |g|$ and $\varphi=\varphi_{\alpha}+\log \left|f_{\alpha}\right|$.

Lemma 3.2. Let $(X, \omega),\left(L_{p}, h_{p}\right)$ verify assumptions $(A)-(B)$, and let $F=\mathcal{O}_{\widetilde{X}}(-\Sigma), \theta, \Omega=$ $C \pi^{*} \omega+c_{1}(F, \theta)$ be as in Lemma 2.2. Then there exist $\alpha \in(0,1), b_{p} \in \mathbb{N}$, and singular Hermitian metrics $\widetilde{h}_{p}$ on $\left.\pi^{*} L_{p}\right|_{\tilde{X} \backslash \Sigma}$ such that $a_{p} \geq C b_{p}, b_{p} \rightarrow \infty$ and $b_{p} / A_{p} \rightarrow 0$ as $p \rightarrow \infty$, $\widetilde{h}_{p} \geq \alpha^{b_{p}} \pi^{*} h_{p}$ and $c_{1}\left(\pi^{*} L_{p}, \widetilde{h}_{p}\right) \geq b_{p} \Omega$ on $\widetilde{X} \backslash \Sigma$. Moreover, for every open relatively compact subset $\widetilde{U}$ of $\widetilde{X} \backslash \Sigma$ there exists a constant $\beta_{\widetilde{U}}>1$ such that $\widetilde{h}_{p} \leq \beta_{\widetilde{U}}^{b_{p}} \pi^{*} h_{p}$ on $\widetilde{U}$.

Proof. If $h^{\prime}, h^{\prime \prime}$ are singular Hermitian metrics on some holomorphic line bundle $G$ then by $h^{\prime} \geq c h^{\prime \prime}$ we mean that $|e|_{h^{\prime}}^{2} \geq c|e|_{h^{\prime \prime}}^{2}$ for any $e \in G$. Consider the line bundles $E_{p}=$ $\pi^{*} L_{p} \otimes F^{b_{p}}$ with metrics $\theta_{p}=\pi^{*} h_{p} \otimes \theta^{b_{p}}$, where $b_{p} \in \mathbb{N}$. Then

$$
c_{1}\left(E_{p}, \theta_{p}\right)=\pi^{*} c_{1}\left(L_{p}, h_{p}\right)+b_{p} c_{1}(F, \theta) \geq a_{p} \pi^{*} \omega+b_{p} c_{1}(F, \theta) \geq b_{p} \Omega,
$$


provided that $a_{p} \geq C b_{p}$. Since $F$ is trivial on $\widetilde{X} \backslash \Sigma$ we have $\left.\left.\pi^{*} L_{p}\right|_{\tilde{X} \backslash \Sigma} \cong E_{p}\right|_{\tilde{X} \backslash \Sigma}$ and we can find a smooth weight $\varphi$ of $\theta$ on $\widetilde{X} \backslash \Sigma$ by setting $|f|_{\theta}^{2}=e^{-2 \varphi}$, where $f$ is a holomorphic frame of $F$ on $\widetilde{X} \backslash \Sigma$. Let $\widetilde{h}_{p}$ be the metric of $\left.\pi^{*} L_{p}\right|_{\widetilde{X} \backslash \Sigma}$ defined by $\widetilde{h}_{p}=e^{-2 b_{p} \varphi^{*}} \pi^{*} h_{p}$. Then $c_{1}\left(\pi^{*} L_{p}, \widetilde{h}_{p}\right)=c_{1}\left(E_{p}, \theta_{p}\right) \geq b_{p} \Omega$ on $\tilde{X} \backslash \Sigma$. Since $\varphi \in \mathscr{C}^{\infty}(\tilde{X} \backslash \Sigma)$ and by Lemma 3.1 $\varphi(y) \rightarrow-\infty$ as $y \rightarrow \Sigma$, it follows that there exists $\alpha \in(0,1)$ such that $e^{-2 \varphi} \geq \alpha$ on $\widetilde{X} \backslash \Sigma$. Moreover, if $\widetilde{U}$ is an open relatively compact subset of $\widetilde{X} \backslash \Sigma$, there exists $\beta_{\widetilde{U}}>1$ such that $e^{-2 \varphi} \leq \beta_{\widetilde{U}}$ on $\widetilde{U}$. These imply that $\widetilde{h}_{p} \geq \alpha^{b_{p}} \pi^{*} h_{p}$ on $\widetilde{X} \backslash \Sigma$ and $\widetilde{h}_{p} \leq \beta_{\widetilde{U}}^{b_{p}} \pi^{*} h_{p}$ on $\widetilde{U}$. The lemma follows if we choose $b_{p} \in \mathbb{N}$ such that $a_{p} \geq C b_{p}, b_{p} \rightarrow \infty$ and $b_{p} / A_{p} \rightarrow 0$ as $p \rightarrow \infty$.

Proof of Theorem 1.1 Note that (ii) follows at once from (i) by using (7). The proof of (i) will be done in two steps.

Step 1. We show here that $\frac{1}{A_{p}} \log P_{p} \rightarrow 0$ as $p \rightarrow \infty$ in $L_{\text {loc }}^{1}\left(X_{\text {reg }}, \omega^{n}\right)$. Fix $x \in X_{\text {reg }}$, $W \Subset X_{\text {reg }}$ a contractible Stein coordinate neighborhood of $x, r_{0}>0$ such that the (closed) ball $V:=B\left(x, 2 r_{0}\right) \subset W$, and set $U:=B\left(x, r_{0}\right)$.

Note that on a Stein manifold $M$ we have $H^{1}\left(M, \mathscr{O}^{*}\right) \cong H^{2}(M, \mathbb{Z})$ due to Cartan's theorem B (see e.g. [H1, p. 201]), thus any holomorphic line bundle $L$ over a Stein contractible manifold $\Omega$ is holomorphically trivial (this is due to [Oka], and is of course a special case of the Oka-Grauert principle). Thus there exist local holomorphic frames $e_{p}^{\prime}$ : $W \longrightarrow L_{p}$, for all $p$. Let $\psi_{p}^{\prime}$ be the corresponding psh weights of $h_{p}$ on $W,\left|e_{p}^{\prime}\right|_{h_{p}}^{2}=e^{-2 \psi_{p}^{\prime}}$. The sequence of currents $\left\{\frac{1}{A_{p}} c_{1}\left(L_{p}, h_{p}\right)\right\}$ has uniformly bounded mass, so it follows from [DS2, Proposition A.16] (see also [DNS]) that there exist psh functions $\psi_{p}$ on int $V$ such that $d d^{c} \psi_{p}=c_{1}\left(L_{p}, h_{p}\right)$ and the sequence $\left\{\frac{1}{A_{p}} \psi_{p}\right\}$ is bounded, hence relatively compact [H2, Theorem 3.2.12], in $L_{l o c}^{1}\left(\right.$ int $\left.V, \omega^{n}\right)$. Since $\psi_{p}^{\prime}-\psi_{p}$ is pluriharmonic, we have $\psi_{p}^{\prime}-\psi_{p}=$ $\operatorname{Re} f_{p}$ for some function $f_{p} \in \mathcal{O}(\operatorname{int} V)$. Considering the local frames $e_{p}=e^{f_{p}} e_{p}^{\prime}$ of $\left.L_{p}\right|_{\operatorname{int} V}$, we obtain

$$
\left|e_{p}\right|_{h_{p}}^{2}=e^{2 \operatorname{Re} f_{p}}\left|e_{p}^{\prime}\right|_{h_{p}}^{2}=e^{-2 \psi_{p}},
$$

i.e., $\psi_{p}$ is the psh weight of $h_{p}$ relative to the frame $e_{p}$.

Let $\left\{b_{p}\right\}$ be as in Lemma 3.2. We prove that there exist $C^{\prime}>1$ and $p_{0} \in \mathbb{N}$ such that

$$
-\frac{b_{p} \log C^{\prime}}{A_{p}} \leq \frac{\log P_{p}(z)}{A_{p}} \leq \frac{\log \left(C^{\prime} r^{-2 n}\right)}{A_{p}}+\frac{2}{A_{p}}\left(\max _{B(z, r)} \psi_{p}-\psi_{p}(z)\right)
$$

holds for all $p>p_{0}, 0<r<r_{0}$, and $z \in U$ with $\psi_{p}(z)>-\infty$. The upper bound in (14) is proved exactly as the corresponding upper bound from the proof of [CM1, Theorem 5.1]. For the lower bound, we show that there exist $c \in(0,1)$ and $p_{0} \in \mathbb{N}$ such that if $p>p_{0}$ and $z \in U, \psi_{p}(z)>-\infty$, then there exists a section $S_{z, p} \in H_{(2)}^{0}\left(X, L_{p}\right)$ verifying $S_{z, p}(z) \neq 0$ and

$$
c^{b_{p}}\left\|S_{z, p}\right\|_{p}^{2} \leq\left|S_{z, p}(z)\right|_{h_{p}}^{2} .
$$


This implies that

$$
\frac{1}{A_{p}} \log P_{p}(z)=\frac{1}{A_{p}} \max _{\|S\|_{p}=1} \log |S(z)|_{h_{p}}^{2} \geq \frac{b_{p} \log c}{A_{p}} .
$$

To prove (15) we work on $\tilde{X} \backslash \Sigma$ and recall that $\pi: \tilde{X} \backslash \Sigma \longrightarrow X_{\text {reg }}$ is a biholomorphism. Let $\Omega \geq \pi^{*} \omega$ be the Kähler form on $\widetilde{X}$ constructed in Lemma 2.2, and $b_{p}, \widetilde{h}_{p}$ be as in Lemma 3.2. Then $b_{p} \rightarrow \infty$ and

$$
c_{1}\left(\left.\pi^{*} L_{p}\right|_{\tilde{X} \backslash \Sigma}, \widetilde{h}_{p}\right) \geq b_{p} \Omega \text { on } \widetilde{X} \backslash \Sigma .
$$

Since $\Omega$ is a Kähler form on $\widetilde{X}$ we have $\operatorname{Ric}_{\Omega} \geq-2 \pi B \Omega$ on $\tilde{X}$, for some constant $B>0$. Moreover, since $\widetilde{X}$ is a compact Kähler manifold, $\widetilde{X} \backslash \Sigma$ has a complete Kähler metric (see [D2], [O]). Repeating the argument in the proof of [CM1, Theorem 5.1] (see also [CM2, Theorems 4.2 and 4.3]) one applies the Ohsawa-Takegoshi extension theorem [OT] and then solves a suitable $\bar{\partial}$-equation using Theorem [2.5, as in [D5, Proposition 3.1], [D7, Section 9], to show the following: there exist $C^{\prime \prime}>1, p_{0} \in \mathbb{N}$, such that if $p>p_{0}$ and $\widetilde{z} \in \pi^{-1}(U), \psi_{p} \circ \pi(\widetilde{z})>-\infty$, then there exists a section $\widetilde{S} \in H^{0}\left(\widetilde{X} \backslash \Sigma, \pi^{*} L_{p}\right)$ verifying $\widetilde{S}(\widetilde{z}) \neq 0$ and

$$
\int_{\widetilde{X} \backslash \Sigma}|\widetilde{S}|_{\widetilde{h}_{p}}^{2} \frac{\Omega^{n}}{n !} \leq C^{\prime \prime}|\widetilde{S}(\widetilde{z})|_{\widetilde{h}_{p}}^{2}
$$

It is important to recall here that the weight of $\widetilde{h}_{p}$ near $\widetilde{z}$ is the sum of $\psi_{p} \circ \pi$ and a smooth function. By Lemma 3.2 we have for all $p$ that $\widetilde{h}_{p} \geq \alpha^{b_{p}} \pi^{*} h_{p}$ on $\tilde{X} \backslash \Sigma$ and $\widetilde{h}_{p} \leq \beta^{b_{p}} \pi^{*} h_{p}$ on $\pi^{-1}(U)$, for some constant $\beta>1$. Since $\Omega \geq \pi^{*} \omega$ these imply that

$$
\alpha^{b_{p}} \int_{\widetilde{X} \backslash \Sigma}|\widetilde{S}|_{\pi^{*} h_{p}}^{2} \frac{\pi^{*} \omega^{n}}{n !} \leq C^{\prime \prime} \beta^{b_{p}}|\widetilde{S}(\widetilde{z})|_{\pi^{*} h_{p}}^{2} .
$$

Fix a constant $c \in(0,1)$ with $C^{\prime \prime} c^{b_{p}} \leq(\alpha / \beta)^{b_{p}}$ for all $p$ and let $S_{z, p}$, where $z=\pi(\widetilde{z})$, be the section of $\left.L_{p}\right|_{X_{\text {reg }}}$ induced by $\widetilde{S}$. Since $X$ is normal it follows that $S_{z, p}$ extends to a holomorphic section of $L_{p}$ on $X$. Moreover, $S_{z, p}(z) \neq 0$ and (15) holds for $S_{z, p}$ and $c$. The proof of (14) is now complete.

To conclude Step 1, it suffices to show that every subsequence of $\left\{\frac{1}{A_{p}} \log P_{p}\right\}$ has a subsequence convergent to 0 in $L^{1}\left(U, \omega^{n}\right)$. Without loss of generality, we prove that $\left\{\frac{1}{A_{p}} \log P_{p}\right\}$ has a subsequence convergent to 0 in $L^{1}\left(U, \omega^{n}\right)$. Since $\left\{\frac{1}{A_{p}} \psi_{p}\right\}$ is locally uniformly upper bounded in int $V$ and relatively compact in $L_{l o c}^{1}\left(\right.$ int $\left.V, \omega^{n}\right)$, there exists a subsequence $\left\{\psi_{p_{j}}\right\}$ so that

$$
\frac{1}{A_{p_{j}}} \psi_{p_{j}} \rightarrow \psi=\left(\limsup \frac{1}{A_{p_{j}}} \psi_{p_{j}}\right)^{*}
$$

in $L_{l o c}^{1}\left(\right.$ int $\left.V, \omega^{n}\right)$ and a.e. on $\operatorname{int} V$, where $\psi \in P S H(\operatorname{int} V)$. Moreover, by the Hartogs lemma,

$$
\lim \sup \frac{1}{A_{p_{j}}} \max _{B(z, r)} \psi_{p_{j}} \leq \max _{B(z, r)} \psi,
$$


for each $z \in U$ and $r<r_{0}$ (see e.g., [H2, Theorem 3.2.13]). Letting $p_{j} \rightarrow \infty$ in (14) we get, since $b_{p} / A_{p} \rightarrow 0$, that

$$
0 \leq \liminf \frac{\log P_{p_{j}}(z)}{A_{p_{j}}} \leq \limsup \frac{\log P_{p_{j}}(z)}{A_{p_{j}}} \leq 2\left(\max _{B(z, r)} \psi-\psi(z)\right)
$$

for a.e. $z \in U$ and every $r<r_{0}$. Letting $r \searrow 0$ and using the upper semicontinuity of $\psi$ we deduce that $\frac{1}{A_{p_{j}}} \log P_{p_{j}} \rightarrow 0$ a.e. on $U$. Since $\left\{\frac{1}{A_{p}} \psi_{p}\right\}$ is locally uniformly upper bounded in int $V$ it follows by (14) that there exists a constant $C^{\prime \prime}>0$ such that

$$
\left|\frac{1}{A_{p_{j}}} \log P_{p_{j}}\right| \leq C^{\prime \prime}-\frac{2}{A_{p_{j}}} \psi_{p_{j}} \text { a.e. on } U \text {. }
$$

As $\psi_{p_{j}}, \psi \in L^{1}\left(U, \omega^{n}\right), \frac{1}{A_{p_{j}}} \psi_{p_{j}} \rightarrow \psi$ a.e. on $U$ and in $L^{1}\left(U, \omega^{n}\right)$, and since $\frac{1}{A_{p_{j}}} \log P_{p_{j}} \rightarrow$ 0 a.e. on $U$, the generalized Lebesgue dominated convergence theorem implies that $\frac{1}{A_{p_{j}}} \log P_{p_{j}} \rightarrow 0$ in $L^{1}\left(U, \omega^{n}\right)$.

Step 2. To complete the proof of $(i)$ we show here that there exists a compact set $K \subset X$ such that $X_{\text {sing }} \subset$ int $K$ and

$$
\frac{1}{A_{p}} \int_{K}\left|\log P_{p}\right| \omega^{n} \rightarrow 0 \text { as } p \rightarrow \infty .
$$

Let $H_{(2)}^{0}\left(\widetilde{X}, \pi^{*} L_{p}\right)$ be the Bergman spaces from Lemma 2.1. We note that there exists $M>0$ such that

$$
\int_{\widetilde{X}} c_{1}\left(\pi^{*} L_{p}, \pi^{*} h_{p}\right) \wedge \Omega^{n-1} \leq M A_{p}, \quad \forall p \geq 1 .
$$

Indeed, since $c_{1}\left(L_{p}, h_{p}\right), T_{0} \in \mathscr{T}(X)$ and $c_{1}\left(L_{p}, h_{p}\right) \leq A_{p} T_{0}$, we have $c_{1}\left(\pi^{*} L_{p}, \pi^{*} h_{p}\right)=$ $\pi^{*} c_{1}\left(L_{p}, h_{p}\right) \leq A_{p} \pi^{*} T_{0}$. This yields (16) with $M=\int_{\tilde{X}} \pi^{*} T_{0} \wedge \Omega^{n-1}$.

We fix now $y \in \Sigma$ and $\widetilde{W}$ an open neighborhood of $y$ in $\widetilde{X}$ biholomorphic to a ball in $\mathbb{C}^{n}$. Using (16) and repeating an argument from Step 1, we find holomorphic frames $\widetilde{e}_{p}$ of $\left.\pi^{*} L_{p}\right|_{\widetilde{W}}$ for which the corresponding psh weights $\widetilde{\psi}_{p}$ of $\pi^{*} h_{p}$ are such that $\left\{\frac{1}{A_{p}} \widetilde{\psi}_{p}\right\}$ is bounded in $L_{\text {loc }}^{1}\left(\widetilde{W}, \Omega^{n}\right)$, hence locally uniformly upper bounded on $\widetilde{W}$ and relatively compact in $L_{l o c}^{1}\left(\widetilde{W}, \Omega^{n}\right)$. If $\left\{\widetilde{S}_{j}^{p}: 1 \leq j \leq d_{p}\right\}$ is an orthonormal basis of $H_{(2)}^{0}\left(\widetilde{X}, \pi^{*} L_{p}\right)$ we write $\widetilde{S}_{j}^{p}=\widetilde{s}_{j}^{p} \widetilde{e}_{p}$ and let $\widetilde{v}_{p}=\frac{1}{2} \log \left(\sum_{j=1}^{d_{p}}\left|\widetilde{s}_{j}^{p}\right|^{2}\right) \in P S H(\widetilde{W})$. By Lemma 2.1, $P_{p} \circ \pi$ is the Bergman kernel function of $H_{(2)}^{0}\left(\widetilde{X}, \pi^{*} L_{p}\right)$, hence

$$
\frac{1}{A_{p}} \widetilde{v}_{p}-\frac{1}{A_{p}} \widetilde{\psi}_{p}=\frac{1}{2 A_{p}} \log P_{p} \circ \pi .
$$

We claim that $\frac{1}{A_{p}} \log P_{p} \circ \pi \rightarrow 0$ in $L_{l o c}^{1}\left(\widetilde{W}, \Omega^{n}\right)$. As in Step 1, it suffices to produce a subsequence with this property. Since $\left\{\frac{1}{A_{p}} \widetilde{\psi}_{p}\right\}$ is relatively compact in $L_{l o c}^{1}\left(\widetilde{W}, \Omega^{n}\right)$ there is a subsequence $\left\{\frac{1}{A_{p_{j}}} \widetilde{\psi}_{p_{j}}\right\}$ convergent in $L_{l o c}^{1}\left(\widetilde{W}, \Omega^{n}\right)$ to a psh function $\widetilde{\psi}$ on $\widetilde{W}$. By Step $1, \frac{1}{A_{p}} \log P_{p} \rightarrow 0$ as $p \rightarrow \infty$ in $L_{\text {loc }}^{1}\left(X_{\text {reg }}, \omega^{n}\right)$, so as $\pi: \widetilde{X} \backslash \Sigma \longrightarrow X_{\text {reg }}$ is biholomorphic, 
$\frac{1}{A_{p}} \log P_{p} \circ \pi \rightarrow 0$ in $L_{l o c}^{1}\left(\widetilde{W} \backslash \Sigma, \Omega^{n}\right)$. Thus $\frac{1}{A_{p_{j}}} \widetilde{v}_{p_{j}} \rightarrow \widetilde{\psi}$ in $L_{l o c}^{1}\left(\widetilde{W} \backslash \Sigma, \Omega^{n}\right)$. By the argument in the proof of [CM2, Theorem 1.1 (i)], we see that $\left\{\frac{1}{A_{p_{j}}}{\widetilde{v_{j}}}_{p_{j}}\right\}$ is locally uniformly upper bounded in $\widetilde{W}$ and it converges to $\widetilde{\psi}$ in $L_{l o c}^{1}\left(\widetilde{W}, \Omega^{n}\right)$. Hence $\frac{1}{A_{p_{j}}} \log P_{p_{j}} \circ \pi \rightarrow 0$ in $L_{l o c}^{1}\left(\widetilde{W}, \Omega^{n}\right)$, which proves our claim.

Since $y \in \Sigma$ was arbitrary and $\Sigma$ is compact we can find an open set $\widetilde{U} \supset \Sigma$ so that $\frac{1}{A_{p}} \log P_{p} \circ \pi \rightarrow 0$ in $L^{1}\left(\widetilde{U}, \Omega^{n}\right)$. Then we fix a compact set $K \subset X$ such that $X_{\text {sing }} \subset \operatorname{int} K$ and $\pi^{-1}(K) \subset \widetilde{U}$. As $\pi: \widetilde{X} \backslash \Sigma \longrightarrow X_{\text {reg }}$ is biholomorphic and $\pi^{*} \omega \leq \Omega$ we have

$$
\begin{aligned}
\frac{1}{A_{p}} \int_{K}\left|\log P_{p}\right| \omega^{n} & =\frac{1}{A_{p}} \int_{K \cap X_{\mathrm{reg}}}\left|\log P_{p}\right| \omega^{n}=\frac{1}{A_{p}} \int_{\pi^{-1}(K) \backslash \Sigma}\left|\log P_{p} \circ \pi\right| \pi^{*} \omega^{n} \\
& \leq \frac{1}{A_{p}} \int_{\pi^{-1}(K)}\left|\log P_{p} \circ \pi\right| \Omega^{n} \rightarrow 0 \text { as } p \rightarrow \infty .
\end{aligned}
$$

This concludes Step 2 and finishes the proof of Theorem 1.1.

Theorem 1.1 holds under the weaker hypotheses obtained if one replaces the domination assumption (2) by (16), where $\pi: \widetilde{X} \rightarrow X$ is the resolution of singularities fixed in Section 2, The proof goes through without change. Due to the presence of singularities of $X$, it is not clear whether (16) holds true without the domination condition (2), i.e., if the mass of the pull-back currents $\pi^{*} c_{1}\left(L_{p}, h_{p}\right)$ on $\widetilde{X}$ is dominated by the mass of the currents $c_{1}\left(L_{p}, h_{p}\right)$ on $X$, uniformly in $p$.

Proof of Theorem [1.2 We repeat the argument in Step 1 from the previous proof, working directly on $\left(X, L_{p}, h_{p}\right)$ with the Kähler form $\omega$.

Proof of Theorem 1.3 We use methods from [Be, Section 2]. Let us consider a reference cover of $X$ as in Definition 2.6. We fix $x \in X$, so $x \in \Delta^{n}\left(x_{j}, R_{j}\right)$ for some $j$, and we pick coordinates $z$ centered at $x$ as in Lemma 2.7, Let

$$
\varphi_{p}(z)=\varphi_{p}^{\prime}(z)+\widetilde{\varphi}_{p}(z), \quad \varphi_{p}^{\prime}(z)=\sum_{l=1}^{n} \lambda_{l}^{p}\left|z_{l}\right|^{2},
$$

be a weight of $h_{p}$ on $\Delta^{n}(x, R)$ so that $\widetilde{\varphi}_{p}$ verifies (ii) in Lemma 2.7 and let $e_{p}$ be a frame of $L_{p}$ on $U_{j}$ with $\left|e_{p}\right|_{h_{p}}=e^{-\varphi_{p}}$. Finally, let $r_{p} \in(0, R / 2)$ be an arbitrary number which will be specified later.

We begin by estimating the norm of a section $S \in H^{0}\left(X, L_{p}\right)$ at $x$. Writing $S=s e_{p}$, where $s \in \mathcal{O}\left(\Delta^{n}(x, R)\right)$, we obtain by the sub-averaging inequality for psh functions

$$
|S(x)|_{h_{p}}^{2}=|s(0)|^{2} \leq \frac{\int_{\Delta^{n}\left(0, r_{p}\right)}|s|^{2} e^{-2 \varphi_{p}^{\prime}} d m}{\int_{\Delta^{n}\left(0, r_{p}\right)} e^{-2 \varphi_{p}^{\prime}} d m} .
$$

If $C>1$ is the constant from Lemma 2.7, we have

$$
\begin{aligned}
\int_{\Delta^{n}\left(0, r_{p}\right)}|s|^{2} e^{-2 \varphi_{p}^{\prime}} d m & \leq\left(1+C r_{p}^{2}\right) \exp \left(2 \max _{\Delta^{n}\left(0, r_{p}\right)} \widetilde{\varphi}_{p}\right) \int_{\Delta^{n}\left(0, r_{p}\right)}|s|^{2} e^{-2 \varphi_{p}} \frac{\omega^{n}}{n !} \\
& \leq\left(1+C r_{p}^{2}\right) \exp \left(2 C\left\|h_{p}\right\|_{3} r_{p}^{3}\right)\|S\|_{p}^{2} .
\end{aligned}
$$


Set

$$
E(r):=\int_{|\xi| \leq r} e^{-2|\xi|^{2}} d m(\xi)=\frac{\pi}{2}\left(1-e^{-2 r^{2}}\right),
$$

where $d m$ is the Lebesgue measure on $\mathbb{C}$. Since $\lambda_{j}^{p} \geq a_{p}$ we obtain

$$
\frac{E\left(r_{p} \sqrt{a_{p}}\right)^{n}}{\lambda_{1}^{p} \ldots \lambda_{n}^{p}} \leq \int_{\Delta^{n}\left(0, r_{p}\right)} e^{-2 \varphi_{p}^{\prime}} d m \leq \int_{\mathbb{C}^{n}} e^{-2 \varphi_{p}^{\prime}} d m=\frac{(\pi / 2)^{n}}{\lambda_{1}^{p} \ldots \lambda_{n}^{p}} .
$$

Combining these estimates it follows that

$$
|S(x)|_{h_{p}}^{2} \leq \frac{\left(1+C r_{p}^{2}\right) \exp \left(2 C\left\|h_{p}\right\|_{3} r_{p}^{3}\right)}{E\left(r_{p} \sqrt{a_{p}}\right)^{n}} \lambda_{1}^{p} \ldots \lambda_{n}^{p}\|S\|_{p}^{2} .
$$

By taking the supremum in (17) over all $S \in H^{0}\left(X, L_{p}\right)$ with $\|S\|_{p}=1$ we get

$$
\frac{P_{p}(x)}{\lambda_{1}^{p} \ldots \lambda_{n}^{p}} \leq \frac{\left(1+C r_{p}^{2}\right) \exp \left(2 C\left\|h_{p}\right\|_{3} r_{p}^{3}\right)}{E\left(r_{p} \sqrt{a_{p}}\right)^{n}}, \quad \forall r_{p} \in(0, R / 2)
$$

For the lower estimate on $P_{p}$, let $0 \leq \chi \leq 1$ be a cut-off function on $\mathbb{C}^{n}$ with support in $\Delta^{n}(0,2), \chi \equiv 1$ on $\Delta^{n}(0,1)$, and set $\chi_{p}(z)=\chi\left(z / r_{p}\right)$. Then $F=\chi_{p} e_{p}$ is a section of $L_{p}$ and $|F(x)|_{h_{p}}=\left|e_{p}(x)\right|_{h_{p}}=e^{-\varphi_{p}(0)}=1$. We have

$$
\begin{aligned}
\|F\|_{p}^{2} & \leq \int_{\Delta^{n}\left(0,2 r_{p}\right)} e^{-2 \varphi_{p}} \frac{\omega^{n}}{n !} \\
& \leq\left(1+4 C r_{p}^{2}\right) \exp \left(16 C\left\|h_{p}\right\|_{3} r_{p}^{3}\right) \int_{\Delta^{n}\left(0,2 r_{p}\right)} e^{-2 \varphi_{p}^{\prime}} d m \\
& \leq\left(\frac{\pi}{2}\right)^{n} \frac{\left(1+4 C r_{p}^{2}\right) \exp \left(16 C\left\|h_{p}\right\|_{3} r_{p}^{3}\right)}{\lambda_{1}^{p} \ldots \lambda_{n}^{p}}
\end{aligned}
$$

Set $\alpha=\bar{\partial} F$. Since $\left\|\bar{\partial} \chi_{p}\right\|^{2}=\|\bar{\partial} \chi\|^{2} / r_{p}^{2}$, where $\|\bar{\partial} \chi\|$ denotes the maximum of $|\bar{\partial} \chi|$, we obtain as above

$$
\|\alpha\|_{p}^{2}=\int_{\Delta^{n}\left(0,2 r_{p}\right)}\left|\bar{\partial} \chi_{p}\right|^{2} e^{-2 \varphi_{p}} \frac{\omega^{n}}{n !} \leq \frac{\|\bar{\partial} \chi\|^{2}}{r_{p}^{2}}\left(\frac{\pi}{2}\right)^{n} \frac{\left(1+4 C r_{p}^{2}\right) \exp \left(16 C\left\|h_{p}\right\|_{3} r_{p}^{3}\right)}{\lambda_{1}^{p} \ldots \lambda_{n}^{p}} .
$$

Since $a_{p} \rightarrow \infty$ there exists $p_{0} \in \mathbb{N}$ such that for $p>p_{0}$ we can solve the $\bar{\partial}$-equation by Theorem 2.5. We get a smooth section $G$ of $L_{p}$ with $\bar{\partial} G=\alpha=\bar{\partial} F$ and

$$
\|G\|_{p}^{2} \leq \frac{2}{a_{p}}\|\alpha\|_{p}^{2} \leq \frac{2\|\bar{\partial} \chi\|^{2}}{a_{p} r_{p}^{2}}\left(\frac{\pi}{2}\right)^{n} \frac{\left(1+4 C r_{p}^{2}\right) \exp \left(16 C\left\|h_{p}\right\|_{3} r_{p}^{3}\right)}{\lambda_{1}^{p} \ldots \lambda_{n}^{p}} .
$$

Since $F=e_{p}$ is holomorphic on $\Delta^{n}\left(0, r_{p}\right), G$ is holomorphic on $\Delta^{n}\left(0, r_{p}\right)$ as $\bar{\partial} G=\bar{\partial} F=0$ there. So the estimate (17) applies to $G$ on $\Delta^{n}\left(0, r_{p}\right)$ and gives

$$
\begin{aligned}
|G(x)|_{h_{p}}^{2} & \leq \frac{\left(1+C r_{p}^{2}\right) \exp \left(2 C\left\|h_{p}\right\|_{3} r_{p}^{3}\right)}{E\left(r_{p} \sqrt{a_{p}}\right)^{n}} \lambda_{1}^{p} \ldots \lambda_{n}^{p}\|G\|_{p}^{2} \\
& \leq \frac{2\|\bar{\partial} \chi\|^{2}}{a_{p} r_{p}^{2} E\left(r_{p} \sqrt{a_{p}}\right)^{n}}\left(\frac{\pi}{2}\right)^{n}\left(1+4 C r_{p}^{2}\right)^{2} \exp \left(18 C\left\|h_{p}\right\|_{3} r_{p}^{3}\right)
\end{aligned}
$$


Let $S=F-G \in H^{0}\left(X, L_{p}\right)$. Then

$$
\begin{aligned}
|S(x)|_{h_{p}}^{2} & \geq\left(|F(x)|_{h_{p}}-|G(x)|_{h_{p}}\right)^{2}=\left(1-|G(x)|_{h_{p}}\right)^{2} \\
& \geq\left[1-\left(\frac{\pi}{2}\right)^{n / 2} \frac{\sqrt{2}\|\bar{\partial} \chi\|\left(1+4 C r_{p}^{2}\right)}{r_{p} \sqrt{a_{p}} E\left(r_{p} \sqrt{a_{p}}\right)^{n / 2}} \exp \left(9 C\left\|h_{p}\right\|_{3} r_{p}^{3}\right)\right]^{2}=: K_{1}\left(r_{p}\right) .
\end{aligned}
$$

Moreover, by (19) and (20)

$$
\|S\|_{p}^{2} \leq\left(\|F\|_{p}+\|G\|_{p}\right)^{2} \leq\left(\frac{\pi}{2}\right)^{n} \frac{K_{2}\left(r_{p}\right)}{\lambda_{1}^{p} \ldots \lambda_{n}^{p}},
$$

where

Therefore

$$
K_{2}\left(r_{p}\right)=\left(1+4 C r_{p}^{2}\right) \exp \left(16 C\left\|h_{p}\right\|_{3} r_{p}^{3}\right)\left(1+\frac{\sqrt{2}\|\bar{\partial} \chi\|}{r_{p} \sqrt{a_{p}}}\right)^{2}
$$

$$
P_{p}(x) \geq \frac{|S(x)|_{h_{p}}^{2}}{\|S\|_{p}^{2}} \geq \frac{\lambda_{1}^{p} \ldots \lambda_{n}^{p}}{\left(\frac{\pi}{2}\right)^{n}} \frac{K_{1}\left(r_{p}\right)}{K_{2}\left(r_{p}\right)} .
$$

Note that at $x, \omega_{x}=\frac{i}{2} \sum_{j=1}^{n} d z_{j} \wedge d \bar{z}_{j}, c_{1}\left(L_{p}, h_{p}\right)_{x}=d d^{c} \varphi_{p}(0)=\frac{i}{\pi} \sum_{j=1}^{n} \lambda_{j}^{p} d z_{j} \wedge d \bar{z}_{j}$, thus

$$
\frac{c_{1}\left(L_{p}, h_{p}\right)_{x}^{n}}{\omega_{x}^{n}}=\left(\frac{2}{\pi}\right)^{n} \lambda_{1}^{p} \ldots \lambda_{n}^{p} .
$$

By (18) and (21) we conclude that

$$
\frac{K_{1}\left(r_{p}\right)}{K_{2}\left(r_{p}\right)} \leq P_{p}(x) \frac{\omega_{x}^{n}}{c_{1}\left(L_{p}, h_{p}\right)_{x}^{n}} \leq K_{3}\left(r_{p}\right)
$$

holds for every $x \in X, r_{p}<R / 2$ and $p>p_{0}$, where

$$
K_{3}\left(r_{p}\right)=\left(\frac{\pi / 2}{E\left(r_{p} \sqrt{a_{p}}\right)}\right)^{n}\left(1+C r_{p}^{2}\right) \exp \left(2 C\left\|h_{p}\right\|_{3} r_{p}^{3}\right) .
$$

By (5) we have that $\varepsilon_{p}=\left\|h_{p}\right\|_{3}^{1 / 3} a_{p}^{-1 / 2} \rightarrow 0$. We set

$$
r_{p}:=\varepsilon_{p}^{1 / 3}\left\|h_{p}\right\|_{3}^{-1 / 3}=\varepsilon_{p}^{-2 / 3} a_{p}^{-1 / 2}, \text { so }\left\|h_{p}\right\|_{3} r_{p}^{3}=\varepsilon_{p}, r_{p} \sqrt{a_{p}}=\varepsilon_{p}^{-2 / 3} .
$$

As $\left\|h_{p}\right\|_{3} \geq 1$, we have $r_{p} \leq \varepsilon_{p}^{1 / 3}$, thus $r_{p} \rightarrow 0$ as $p \rightarrow \infty$. With this choice for $r_{p}$ we obtain

$$
K_{3}\left(r_{p}\right) \leq\left(\frac{\pi / 2}{E\left(\varepsilon_{p}^{-2 / 3}\right)}\right)^{n}\left(1+C \varepsilon_{p}^{2 / 3}\right) \exp \left(2 C \varepsilon_{p}\right) \leq 1+C^{\prime} \varepsilon_{p}^{2 / 3}, \frac{K_{1}\left(r_{p}\right)}{K_{2}\left(r_{p}\right)} \geq 1-C^{\prime} \varepsilon_{p}^{2 / 3},
$$

where $C^{\prime}>0$ is a constant depending only on the reference cover. Therefore (6) follows from (22) and the proof is complete.

Remark 3.3. Theorem 1.3 shows that

$$
\lim _{p \rightarrow \infty} P_{p}(x) \frac{\omega_{x}^{n}}{c_{1}\left(L_{p}, h_{p}\right)_{x}^{n}}=1 \text {, uniformly on } X \text {. }
$$

This is a generalization of the asymptotic expansion of the Bergman kernel [Ca, DLM], HsM, MM1, MM2, R, T1, Z] for $\left(L_{p}, h_{p}\right)=\left(L^{p}, h^{p}\right)$, where $(L, h)$ is a positive line bundle 
with smooth metric $h$. Indeed, if $\left(L_{p}, h_{p}\right)=\left(L^{p}, h^{p}\right)$, we have $a_{p}=p$ and $\left\|h_{p}\right\|_{3} \leq C_{h} p$, where $C_{h}$ is a constant depending only on $h$ and the reference cover. Hence

$$
\varepsilon_{p}=\left\|h_{p}\right\|_{3}^{1 / 3} a_{p}^{-1 / 2} \leq C_{h}^{1 / 3} p^{-1 / 6},
$$

so condition (5) is fulfilled. Estimate (6) yields

$$
\left|\frac{P_{p}(x)}{p^{n}} \frac{\omega_{x}^{n}}{c_{1}(L, h)_{x}^{n}}-1\right| \leq \frac{C C_{h}^{2 / 9}}{p^{1 / 9}},
$$

hence

$$
\left|P_{p}(x)-b_{0}(x) p^{n}\right| \leq C^{\prime} p^{n-\frac{1}{9}}, \text { where } b_{0}(x):=\frac{c_{1}(L, h)_{x}^{n}}{\omega_{x}^{n}} .
$$

By the above mentioned papers there exists $C>0$ such that $\left|P_{p}(x)-b_{0}(x) p^{n}\right| \leq C p^{n-1}$ on $X$, which gives a sharper estimate of $P_{p}(x)$ than (23)-(24). On the other hand, the method used here can handle the much more general case of sequences of line bundles $\left(L_{p}, h_{p}\right)$ satisfying the minimal hypotheses of Theorem 1.3 .

\section{ZEROS OF HOLOMORPHIC SECTIONS AND APPROXIMATION RESULTS}

We assume as before that $(X, \omega),\left(L_{p}, h_{p}\right) \rightarrow X$ satisfy conditions (A) and (B). Consider the unit sphere $\mathcal{S}^{p} \subset H_{(2)}^{0}\left(X, L_{p}\right), d_{p}=\operatorname{dim} H_{(2)}^{0}\left(X, L_{p}\right)$. We identify the unit sphere $\mathcal{S}^{p}$ to the unit sphere $\mathbf{S}^{2 d_{p}-1}$ in $\mathbb{C}^{d_{p}}$ by

$$
a=\left(a_{1}, \ldots, a_{d_{p}}\right) \in \mathbf{S}^{2 d_{p}-1} \longmapsto S_{a}=\sum_{j=1}^{d_{p}} a_{j} S_{j}^{p} \in \mathcal{S}^{p},
$$

and we let $\lambda_{p}$ be the probability measure on $\mathcal{S}^{p}$ induced by the normalized surface measure on $\mathbf{S}^{2 d_{p}-1}$, denoted also by $\lambda_{p}$ (i.e., $\lambda_{p}\left(\mathbf{S}^{2 d_{p}-1}\right)=1$ ). Consider the probability space $\mathcal{S}_{\infty}=\prod_{p=1}^{\infty} \mathcal{S}^{p}$ endowed with the probability measure $\lambda_{\infty}=\prod_{p=1}^{\infty} \lambda_{p}$. We denote by $[\sigma=0]$ the current of integration (with multiplicities) over the zero set of a nontrivial section $\sigma \in H_{(2)}^{0}\left(X, L_{p}\right)$.

Theorem 4.1. Let $(X, \omega),\left(L_{p}, h_{p}\right), p \geq 1$, verify assumptions $(A)-(B)$ and assume that $\sum_{p=1}^{\infty} \frac{1}{A_{p}^{2}}<\infty$. Then for $\lambda_{\infty}$-a.e. sequence $\left\{\sigma_{p}\right\}_{p \geq 1} \in \mathcal{S}_{\infty}$ we have in the weak sense of currents on $X$ that

$$
\lim _{p \rightarrow \infty} \frac{1}{A_{p}}\left(\left[\sigma_{p}=0\right]-c_{1}\left(L_{p}, h_{p}\right)\right)=0 .
$$

Moreover, if $\lim _{p \rightarrow \infty} \frac{1}{A_{p}} c_{1}\left(L_{p}, h_{p}\right)=T$ for some positive closed current $T$ of bidegree $(1,1)$ on $X$, then for $\lambda_{\infty}$-a.e. sequence $\left\{\sigma_{p}\right\}_{p \geq 1} \in \mathcal{S}_{\infty}$,

$$
\lim _{p \rightarrow \infty} \frac{1}{A_{p}}\left[\sigma_{p}=0\right]=T \text { weakly on } X \text {. }
$$


Proof. The arguments in [SZ1, SZ2] (see also [CM2, Section 5.2]; in all these papers $A_{p}=p$ ) imply that for $\lambda_{\infty}$-a.e. sequence $\left\{\sigma_{p}\right\}_{p \geq 1} \in \mathcal{S}_{\infty}$,

$$
\lim _{p \rightarrow \infty} \frac{1}{A_{p}}\left(\left[\sigma_{p}=0\right]-\gamma_{p}\right)=0
$$

weakly in the sense of currents on $X$. Indeed, by working with a countable set of test forms and since

$$
\int_{X}\left[\sigma_{p}=0\right] \wedge \omega^{n-1}=\int_{X} \gamma_{p} \wedge \omega^{n-1}=\int_{X} c_{1}\left(L_{p}, h_{p}\right) \wedge \omega^{n-1}=A_{p},
$$

it suffices to show that, for a fixed test form $\theta$, one has

$$
\lim _{p \rightarrow \infty} \frac{1}{A_{p}}\left\langle\left[\sigma_{p}=0\right]-\gamma_{p}, \theta\right\rangle=0,
$$

for $\lambda_{\infty}$-a.e. $\sigma=\left\{\sigma_{p}\right\}_{p \geq 1} \in \mathcal{S}_{\infty}$. Let

$$
Y_{p}: \mathcal{S}_{\infty} \longrightarrow \mathbb{C}, \quad Y_{p}(\sigma)=\frac{1}{A_{p}}\left\langle\left[\sigma_{p}=0\right]-\gamma_{p}, \theta\right\rangle .
$$

The calculations in [SZ1, Sec. 3.1-3.3] show that

$$
\int_{\mathcal{S}_{\infty}} Y_{p} d \lambda_{\infty}=0, \quad \int_{\mathcal{S}_{\infty}}\left|Y_{p}\right|^{2} d \lambda_{\infty} \leq \frac{A C_{\theta}}{A_{p}^{2}} \text {, with } A=\frac{1}{\pi^{2}} \int_{\mathbb{C}^{2}}\left(\log \left|z_{1}\right|\right)^{2} e^{-\left|z_{1}\right|^{2}-\left|z_{2}\right|^{2}} d z
$$

where $d z$ is the Lebesgue measure on $\mathbb{C}^{2}$, and $C_{\theta}$ is a constant depending only on $\theta$. Then (25) follows since

$$
\int_{\mathcal{S}_{\infty}}\left(\sum_{p=1}^{\infty}\left|Y_{p}\right|^{2}\right) d \lambda_{\infty} \leq A C_{\theta} \sum_{p=1}^{\infty} \frac{1}{A_{p}^{2}}<+\infty .
$$

We now conclude by Theorem 1.1 .

We show next that equidistribution results hold not only for the zeros of random sequences of holomorphic sections but also for the logarithms of their pointwise norms.

Theorem 4.2. Let $(X, \omega),\left(L_{p}, h_{p}\right), p \geq 1$, verify assumptions $(A)$-(B) and assume that

$$
\liminf _{p \rightarrow \infty} \frac{\log d_{p}}{A_{p}}=0 .
$$

Then there exists an increasing sequence of natural numbers $\left\{p_{j}\right\}_{j \geq 1}$ such that for $\lambda_{\infty}$-a.e. sequence $\left\{\sigma_{p}\right\}_{p \geq 1} \in \mathcal{S}_{\infty}$ we have

$$
\lim _{j \rightarrow \infty} \frac{\log \left|\sigma_{p_{j}}\right| h_{h_{j}}}{A_{p_{j}}}=0 \text { in } L^{1}\left(X, \omega^{n}\right) .
$$

To prove the theorem we need the following elementary lemma whose proof is included for the convenience of the reader. 
Lemma 4.3. If $\mathbf{S}^{2 k-1}$ is the unit sphere in $\mathbb{C}^{k}$ with surface measure $d \mathcal{A}$ and

$$
\mathbf{I}(k):=-\frac{1}{\operatorname{area}\left(\mathbf{S}^{2 k-1}\right)} \int_{\mathbf{S}^{2 k-1}} \log \left|z_{k}\right| d \mathcal{A},
$$

where $z=\left(z_{1}, \ldots, z_{k}\right) \in \mathbb{C}^{k}$, then there exist numbers $a, b>1$ such that

$$
\mathbf{I}(k) \leq a \log k+b, \quad \forall k \geq 1 .
$$

Proof. We use spherical coordinates $\left(\theta_{1}, \ldots, \theta_{2 k-2}, \varphi\right) \in\left[-\frac{\pi}{2}, \frac{\pi}{2}\right]^{2 k-2} \times[0,2 \pi]$ on $\mathbf{S}^{2 k-1}$ such that

$$
z_{k}=\sin \theta_{2 k-3} \cos \theta_{2 k-2}+i \sin \theta_{2 k-2}, \quad d \mathcal{A}=\cos \theta_{1} \cos ^{2} \theta_{2} \ldots \cos ^{2 k-2} \theta_{2 k-2} d \theta_{1} \ldots d \theta_{2 k-2} d \varphi .
$$

We obtain

$$
\mathbf{I}(k)=\frac{J(k)}{2 C_{2 k-2} C_{2 k-3}}, \text { where } C_{k}=\int_{0}^{\pi / 2} \cos ^{k} t d t,
$$

and, after performing the change of variables $x=\sin \theta_{2 k-2}, y=\sin \theta_{2 k-3}$,

$$
J(k)=-\int_{0}^{1} \int_{0}^{1}\left(1-x^{2}\right)^{k-3 / 2}\left(1-y^{2}\right)^{k-2} \log \left(x^{2}+y^{2}-x^{2} y^{2}\right) d x d y .
$$

Since $2 x^{2} y^{2} \leq x^{2}+y^{2}$ for $0 \leq x, y \leq 1$, we have

$$
\begin{aligned}
-\log \left(x^{2}+y^{2}-x^{2} y^{2}\right) & \leq-\log \frac{x^{2}+y^{2}}{2}, \\
\left(1-x^{2}\right)^{k-3 / 2}\left(1-y^{2}\right)^{k-2} & \leq\left(1-x^{2}-y^{2}+x^{2} y^{2}\right)^{k-2} \leq\left(1-\frac{x^{2}+y^{2}}{2}\right)^{k-2},
\end{aligned}
$$

provided that $k \geq 2$. If $D=\left\{(x, y) \in \mathbb{R}^{2}: x \geq 0, y \geq 0, x^{2}+y^{2} \leq 2\right\}$ it follows that

$$
\begin{aligned}
J(k) & \leq-\iint_{D}\left(1-\frac{x^{2}+y^{2}}{2}\right)^{k-2} \log \frac{x^{2}+y^{2}}{2} d x d y \\
& =-\frac{\pi}{2} \int_{0}^{\sqrt{2}} r\left(1-\frac{r^{2}}{2}\right)^{k-2} \log \frac{r^{2}}{2} d r=-\frac{\pi}{2} \int_{0}^{1}(1-t)^{k-2} \log t d t .
\end{aligned}
$$

One shows by induction on $k \geq 2$ that

$$
-\int_{0}^{1}(1-t)^{k-2} \log t d t=\frac{1}{k-1}\left(1+\frac{1}{2}+\ldots+\frac{1}{k-1}\right) \leq \frac{1+\log (k-1)}{k-1} .
$$

The lemma follows since there exist constants $A, B>0$ such that $A \leq C_{k} \sqrt{k} \leq B$ for all $k \geq 1$. Indeed, an integration by parts shows that

$$
C_{k+2}=\frac{k+1}{k+2} C_{k}, \text { so } C_{2 k}=\frac{\pi(2 k) !}{2^{2 k+1}(k !)^{2}}, \quad C_{2 k+1}=\frac{2^{2 k}(k !)^{2}}{(2 k+1) !}, \text { for } k \geq 0 .
$$

By Stirling's formula $k ! \sim(k / e)^{k} \sqrt{k},(2 k) ! \sim(2 k / e)^{2 k} \sqrt{2 k}$, which implies our claim. 
Proof of Theorem 4.2 Using (26) we can find a sequence of integers $p_{j} \nearrow \infty$ such that $\sum_{j=1}^{\infty} \frac{\log d_{p_{j}}}{A_{p_{j}}}<\infty$. We define

$$
Y_{p}: \mathcal{S}_{\infty} \longrightarrow \mathbb{R}, Y_{p}(\sigma)=\frac{1}{A_{p}} \int_{X} \log \frac{\left|\sigma_{p}\right|_{h_{p}}}{\sqrt{P_{p}}} \omega^{n}, \text { where } \sigma=\left\{\sigma_{p}\right\}_{p \geq 1}
$$

By Theorem 1.1 we have $\frac{1}{A_{p}} \log P_{p} \rightarrow 0$ as $p \rightarrow \infty$, in $L^{1}\left(X, \omega^{n}\right)$. Since $\log \frac{\left|\sigma_{p}\right|_{h_{p}}}{\sqrt{P_{p}}} \leq 0$ on $X$ for $\sigma=\left\{\sigma_{p}\right\}_{p \geq 1} \in \mathcal{S}_{\infty}$, it suffices to show that $Y_{p_{j}}(\sigma) \rightarrow 0$ as $j \rightarrow \infty$ for $\lambda_{\infty}$-a.e. $\sigma \in \mathcal{S}_{\infty}$. By Tonelli's theorem we have

$$
\int_{\mathcal{S}_{\infty}} Y_{p}(\sigma) d \lambda_{\infty}=\frac{1}{A_{p}} \int_{\mathcal{S}^{p}}\left(\int_{X} \log \frac{\left|\sigma_{p}\right| h_{p}}{\sqrt{P_{p}}} \omega^{n}\right) d \lambda_{p}=\frac{1}{A_{p}} \int_{X}\left(\int_{\mathcal{S}^{p}} \log \frac{\left|\sigma_{p}\right| h_{p}}{\sqrt{P_{p}}} d \lambda_{p}\right) \omega^{n} .
$$

For a fixed $x \in X$, we write $S_{l}^{p}=s_{l}^{p} e_{p}$ for some holomorphic frame $e_{p}$ of $L_{p}$ near $x$ and we set

$$
u=\left(u_{1}, \ldots, u_{d_{p}}\right), \quad u_{l}=\frac{s_{l}^{p}}{\sqrt{\left|s_{1}^{p}\right|^{2}+\ldots+\left|s_{d_{p}}^{p}\right|^{2}}} .
$$

Then the integral

$$
\int_{\mathcal{S}^{p}} \log \frac{\left|\sigma_{p}(x)\right|_{h_{p}}}{\sqrt{P_{p}(x)}} d \lambda_{p}=\int_{\mathbf{S}^{2 d_{p}-1}} \log |a \cdot u(x)| d \lambda_{p}(a)=-\mathbf{I}\left(d_{p}\right)
$$

is independent of $x$, where $a \cdot u=a_{1} u_{1}+\ldots+a_{d_{p}} u_{d_{p}}$ and $\mathbf{I}(k)$ is defined in Lemma 4.3. Using Lemma 4.3 it follows that

$$
\int_{\mathcal{S}_{\infty}} Y_{p}(\sigma) d \lambda_{\infty} \geq-\frac{a \log d_{p}+b}{A_{p}} \int_{X} \omega^{n}
$$

The definition of the sequence $\left\{p_{j}\right\}_{j \geq 1}$ shows that

$$
\sum_{j=1}^{\infty} \int_{\mathcal{S}_{\infty}} Y_{p_{j}}(\sigma) d \lambda_{\infty}>-\infty
$$

Since $Y_{p} \leq 0$ this implies that $\sum_{j=1}^{\infty} Y_{p_{j}}$ converges in $L^{1}\left(\mathcal{S}_{\infty}, \lambda_{\infty}\right)$, hence $Y_{p_{j}}(\sigma) \rightarrow 0$ as $j \rightarrow \infty$ for $\lambda_{\infty}$-a.e. $\sigma \in \mathcal{S}_{\infty}$.

Let us give two general examples in which condition (26) holds true.

Proposition 4.4. Let $(X, \omega),\left(L_{p}, h_{p}\right), p \geq 1$, verify assumptions (A)-(B) and assume that $X$ is smooth and that each line bundle $L_{p}$ has a continuous metric $h_{p}^{\prime}$ with the following property: every $x \in X$ has a contractible Stein coordinate neighborhood $W_{x}$ on which each metric $h_{p}^{\prime}$ has a weight $\psi_{p}^{\prime}$ such that the family $\left\{\psi_{p}^{\prime} / A_{p}\right\}_{p \geq 1}$ is equicontinuous on $W_{x}$. Then

$$
\lim _{p \rightarrow \infty} \frac{\log \operatorname{dim} H^{0}\left(X, L_{p}\right)}{A_{p}}=0 .
$$


Proof. Let $\varepsilon>0$ and let $P_{p}^{\prime}$ be the Bergman kernel function of the space $H^{0}\left(X, L_{p}\right)$ with respect to the metrics $h_{p}^{\prime}$ and $\omega$. For $x \in X$ fix $r_{x}>0$ so that the (closed) ball $B\left(x, 2 r_{x}\right) \subset$ $W_{x}$ and let $U_{x}=B\left(x, r_{x}\right)$. The proof of the upper bound in (14) works for any metric on $L_{p}$ (see also [CM1, (7)]) and shows that

$$
P_{p}^{\prime}(z) \leq C_{x} r^{-2 n} \exp \left(2 \max _{B(z, r)} \psi_{p}^{\prime}-2 \psi_{p}^{\prime}(z)\right)
$$

for any $p \geq 1, r<r_{x}$ and $z \in U_{x}$, where $C_{x}$ is a constant depending only on $x$. The equicontinuity assumption implies that there exists $r_{1}=r_{1}(x, \varepsilon)<r_{x}$ such that $2 \max _{B\left(z, r_{1}\right)} \psi_{p}^{\prime}-2 \psi_{p}^{\prime}(z) \leq A_{p} \varepsilon$ for all $p \geq 1$ and $z \in U_{x}$, hence $P_{p}^{\prime}(z) \leq C_{x} r_{1}^{-2 n} \exp \left(A_{p} \varepsilon\right)$. A standard compactness argument now shows that there exists a constant $C^{\prime}=C^{\prime}(\varepsilon)>0$ such that $P_{p}^{\prime} \leq C^{\prime} \exp \left(A_{p} \varepsilon\right)$ holds on $X$ for all $p \geq 1$. It follows that

$$
\operatorname{dim} H^{0}\left(X, L_{p}\right)=\int_{X} P_{p}^{\prime} \frac{\omega^{n}}{n !} \leq C^{\prime} \exp \left(A_{p} \varepsilon\right) \int_{X} \frac{\omega^{n}}{n !}, p \geq 1,
$$

which implies the conclusion of the proposition.

The second general example is provided by the class of semi-ample line bundles. Recall that a line bundle $L$ on $X$ is called semi-ample if $L^{k}$ is globally generated for some $k>0$, or, equivalently, the space $H^{0}\left(X, L^{k}\right)$ has no base locus.

Proposition 4.5. Let $(X, \omega),\left(L_{p}, h_{p}\right), p \geq 1$, verify assumptions (A)-(B) and assume that $X$ is smooth and that each line bundle $L_{p}$ is semi-ample. Then there exist an integer $N>0$ and a constant $C>0$ depending only on $\omega$ such that

$$
\operatorname{dim} H^{0}\left(X, L_{p}\right) \leq C A_{p}^{N}, \forall p \geq 1 .
$$

Proof. Since $L_{p}$ is big, $X$ is Moishezon and hence projective since it is Kähler. By the main theorem in $[\mathrm{KM}]$, there exists a polynomial $Q(y, z)$ depending only on $\operatorname{dim} X$ such that for any semi-ample line bundle $L$ on $X$ one has that

$$
\operatorname{dim} H^{0}(X, L) \leq Q\left(\int_{X} c_{1}(L)^{n}, \int_{X} c_{1}(L)^{n-1} \wedge c_{1}(X)\right) .
$$

Lemma 4.6 below implies that there exists a constant $C^{\prime}>0$ depending only on $(X, \omega)$ such that

$$
\int_{X} c_{1}\left(L_{p}\right)^{n} \leq C^{\prime} A_{p}^{n}, \quad \int_{X} c_{1}\left(L_{p}\right)^{n-1} \wedge c_{1}(X) \leq C^{\prime} A_{p}^{n-1} .
$$

The conclusion now follows.

Lemma 4.6. Let $(X, \omega)$ be a compact Kähler manifold of dimension $n$ and let $\beta$ be a real valued closed form of type $(1,1)$ on $X$. Then there exists $C>0$ depending only on $\omega$ and $\beta$ such that for any pseudoeffective class $\alpha \in H^{1,1}(X, \mathbb{R})$ we have

$$
\int_{X} \alpha^{k} \wedge \beta^{n-k} \leq C\|\alpha\|^{k}, k=1, \ldots, n, \text { where }\|\alpha\|=\int_{X} \alpha \wedge \omega^{n-1} .
$$


Proof. If $\|\alpha\|=0$ then $\alpha=0$ since $\alpha$ is pseudoeffective, so we can assume $\|\alpha\|>0$. Let $\theta \in \alpha$ be a smooth form and $T=\theta+d d^{c} \varphi$ be a positive closed current, where $\varphi$ is a $\theta$ psh function. The Lelong numbers $\nu(T, x)<C_{1}\|\alpha\|$ for all $x \in X$, where $C_{1}$ is a constant depending only on $\omega$ (see e.g., [Bo, Lemma 2.5]). Demailly's regularization theorem [D5] shows that there exists a sequence of smooth functions $\varphi_{k} \searrow \varphi$ such $\theta+d d^{c} \varphi_{k} \geq-C_{2} \lambda_{k} \omega$, where $\lambda_{k}$ are continuous functions on $X, \lambda_{k}(x) \searrow \nu(T, x)$ as $k \rightarrow \infty$ for every $x \in X$, and $C_{2}$ is a constant depending only on $\omega$. We fix $k$ so that $\lambda_{k}(x)<C_{1}\|\alpha\|$ for every $x \in X$ and let $R=\theta+d d^{c} \varphi_{k}$ and $R^{\prime}=R+C_{3}\|\alpha\| \omega$, where $C_{3}=C_{1} C_{2}$, so $R^{\prime} \geq 0$. Next we set $\beta^{\prime}=\beta+c \omega$, where $c>0$ is chosen so that $\beta^{\prime} \geq 0$. Since $R^{\prime}, \beta^{\prime} \geq 0$ we obtain

$$
\begin{aligned}
\int_{X} \alpha^{k} \wedge \beta^{n-k} & =\int_{X} R^{k} \wedge \beta^{n-k}=\int_{X}\left(R^{\prime}-C_{3}\|\alpha\| \omega\right)^{k} \wedge\left(\beta^{\prime}-c \omega\right)^{n-k} \\
& \leq \int_{X}\left(R^{\prime}+C_{3}\|\alpha\| \omega\right)^{k} \wedge\left(\beta^{\prime}+c \omega\right)^{n-k} \leq C_{4}\left\|R^{\prime}+C_{3}\right\| \alpha\|\omega\|^{k},
\end{aligned}
$$

where $C_{4}$ is a constant depending only on the Kähler form $\beta^{\prime}+c \omega$ (hence on $\beta$ and $\omega$ ). The lemma follows since

$$
\left\|R^{\prime}+C_{3}\right\| \alpha\|\omega\|=\int_{X}\left(R+2 C_{3}\|\alpha\| \omega\right) \wedge \omega^{n-1}=\left(1+2 C_{3} \int_{X} \omega^{n}\right)\|\alpha\| .
$$

We conclude this section by discussing an application of the above results to the problem of approximation of positive closed currents of bidegree $(1,1)$ on $X$ by currents of integration along analytic hypersurfaces of $X$. Let $\mathscr{A}(X)$ be the space of positive closed currents $T \in \mathcal{D}_{n-1, n-1}^{\prime}(X)$ with the property that there exist a sequence of singular Hermitian holomorphic line bundles $\left\{\left(F_{p}, h^{F_{p}}\right)\right\}_{p \geq 1}$ with $c_{1}\left(F_{p}, h^{F_{p}}\right) \geq 0$ and a sequence of natural numbers $N_{p} \rightarrow \infty$ such that

$$
\lim _{p \rightarrow \infty} \frac{1}{N_{p}} c_{1}\left(F_{p}, h^{F_{p}}\right)=T .
$$

If $X_{\text {sing }} \neq \emptyset$ we require in addition that there exists a current $T_{0} \in \mathscr{T}(X)$ (depending on $T$ ) such that for all $p \geq 1$ we have $\frac{1}{N_{p}} c_{1}\left(F_{p}, h^{F_{p}}\right) \leq T_{0}$.

When $X$ is smooth the space $\mathscr{A}(X)$ is the closure in $\mathcal{D}_{n-1, n-1}^{\prime}(X)$ of the convex cone generated by positive closed integral currents. Recall that a real closed current $T \in$ $\mathcal{D}_{n-1, n-1}^{\prime}(X)$ is called integral if its de Rham cohomology class $[T]$ belongs to $H^{1,1}(X, \mathbb{R}) \cap$ $H^{2}(X, \mathbb{Z})$. A current $T$ is integral if and only if there exists a singular Hermitian holomorphic line bundle $(L, h)$ on $X$ with $c_{1}(L, h)=T$ (see e.g., [MM1, Lemma 2.3.5]).

Theorem 4.7. Let $(X, \omega)$ be a compact normal Kähler space and $(L, h)$ be a singular Hermitian holomorphic line bundle on $X$ such that $c_{1}(L, h) \geq \varepsilon \omega$ for some $\varepsilon>0$. If $T \in \mathscr{A}(X)$ then there exist a sequence of singular Hermitian holomorphic line bundles $\left\{\left(L_{p}, h_{p}\right)\right\}_{p \geq 1}$ with $c_{1}\left(L_{p}, h_{p}\right) \geq 0$ and a sequence of natural numbers $N_{p} \rightarrow \infty$ such that for $\lambda_{\infty}$-a.e. sequence $\left\{\sigma_{p}\right\}_{p \geq 1} \in \mathcal{S}_{\infty}$,

$$
\lim _{p \rightarrow \infty} \frac{1}{N_{p}}\left[\sigma_{p}=0\right]=T \text { weakly on } X
$$


Here the probability space $\left(\mathcal{S}_{\infty}, \lambda_{\infty}\right)$ is associated as above to the sequence $\left\{\left(L_{p}, h_{p}\right)\right\}_{p \geq 1}$.

Proof. Since $T \in \mathscr{A}(X)$ there exist line bundles $\left\{\left(F_{p}, h^{F_{p}}\right)\right\}_{p \geq 1}$ with $c_{1}\left(F_{p}, h^{F_{p}}\right) \geq 0$ and a sequence of natural numbers $N_{p} \rightarrow \infty, p \rightarrow \infty$, such that $\lim _{p \rightarrow \infty} \frac{1}{N_{p}} c_{1}\left(F_{p}, h^{F_{p}}\right)=T$. Moreover, if $X_{\text {sing }} \neq \emptyset$ there exists a current $T_{0} \in \mathscr{T}(X)$ such that $c_{1}\left(F_{p}, h^{F_{p}}\right) \leq N_{p} T_{0}$ for all $p \geq 1$. We can assume without loss of generality that $N_{p} \geq p$; otherwise replace $\left(F_{p}, h^{F_{p}}\right)$ by $\left(F_{p}^{m_{p}},\left(h^{F_{p}}\right)^{m_{p}}\right)$ and $N_{p}$ by $m_{p} N_{p}$, with a convenient $m_{p} \in \mathbb{N}$. We fix a sequence $b_{p} \in \mathbb{N}$ such that $b_{p} \leq N_{p}$ for all $p \geq 1$ and $b_{p} \rightarrow \infty, b_{p} / N_{p} \rightarrow 0$ as $p \rightarrow \infty$. Let

$$
L_{p}=F_{p} \otimes L^{b_{p}}, \quad h_{p}=h^{F_{p}} \otimes h^{b_{p}} .
$$

The conclusion follows from Theorem 4.1 since $c_{1}\left(L_{p}, h_{p}\right) \geq b_{p} c_{1}(L, h) \geq b_{p} \varepsilon \omega$ and

$$
\begin{aligned}
& c_{1}\left(L_{p}, h_{p}\right) \leq N_{p} T_{0}+b_{p} c_{1}(L, h) \leq N_{p}\left(T_{0}+c_{1}(L, h)\right), \\
& \frac{c_{1}\left(L_{p}, h_{p}\right)}{N_{p}} \rightarrow T, \quad \frac{\left\|c_{1}\left(L_{p}, h_{p}\right)\right\|}{N_{p}} \rightarrow\|T\|, \text { as } p \rightarrow \infty .
\end{aligned}
$$

For related approximation results on compact Kähler manifolds we refer to [D7, D1, G, CM3]. We note that Dinh and Sibony [DS1] developed a general method to obtain the asymptotic distribution with speed of convergence of zeros of random sequences of holomorphic sections.

\section{APPLICATIONS}

5.1. Powers of a line bundle. Theorems 1.1, 4.1, 4.2 apply for the sequence $\left(L_{p}, h_{p}\right)=$ $\left(L^{p}, h^{p}\right)$, where $(L, h)$ is a singular Hermitian holomorphic line bundle on $X$ with strictly positive curvature current.

Corollary 5.1. Let $(X, \omega)$ be a compact normal Kähler space and $(L, h)$ be a singular Hermitian holomorphic line bundle on $X$ such that $c_{1}(L, h) \geq \varepsilon \omega$ for some $\varepsilon>0$. Then as $p \rightarrow \infty$ :

(i) $\frac{1}{p} \log P_{p} \rightarrow 0$ in $L^{1}\left(X, \omega^{n}\right)$.

(ii) $\frac{1}{p} \gamma_{p} \rightarrow c_{1}(L, h)$ weakly on $X$.

(iii) $\frac{1}{p}\left[\sigma_{p}=0\right] \rightarrow c_{1}(L, h)$ weakly on $X$, for $\lambda_{\infty}$-a.e. sequence $\left\{\sigma_{p}\right\}_{p \geq 1} \in \mathcal{S}_{\infty}$.

Indeed, assumptions (A) and (B) are satisfied with $a_{p}=p \varepsilon$ and $T_{0}=c_{1}(L, h) /\left\|c_{1}(L, h)\right\|$, where $\left\|c_{1}(L, h)\right\|:=\int_{X} c_{1}(L, h) \wedge \omega^{n-1}$. Moreover $A_{p}=p\left\|c_{1}(L, h)\right\|$.

We consider now the case when the curvature current of the singular metric is not necessarily Kähler.

Corollary 5.2. Let $(L, h)$ be a singular Hermitian holomorphic line bundle on the compact normal Kähler space $(X, \omega)$ such that $c_{1}(L, h) \geq 0$ and assume that $L$ has a singular metric $h_{0}$ with $c_{1}\left(L, h_{0}\right) \geq \varepsilon \omega$ for some $\varepsilon>0$. Let $\left\{n_{p}\right\}_{p \geq 1}$ be a sequence of natural numbers such that

$$
n_{p} \rightarrow \infty \text { and } n_{p} / p \rightarrow 0 \text { as } p \rightarrow \infty
$$


Let

$$
h_{p}=h^{p-n_{p}} \otimes h_{0}^{n_{p}}
$$

and $P_{p}, \gamma_{p}, \mathcal{S}^{p}$ be the Bergman kernel function, Fubini-Study current, and respectively the unit sphere, associated to the spaces $H_{(2)}^{0}\left(X, L_{p}\right)=H_{(2)}^{0}\left(X, L^{p}, h_{p}\right)$. Then, as $p \rightarrow \infty$, we have $\frac{1}{p} \log P_{p} \rightarrow 0$ in $L^{1}\left(X, \omega^{n}\right), \frac{1}{p} \gamma_{p} \rightarrow c_{1}(L, h)$ and $\frac{1}{p}\left[\sigma_{p}=0\right] \rightarrow c_{1}(L, h)$ in the sense of currents on $X$, for $\lambda_{\infty}$-a.e. sequence $\left\{\sigma_{p}\right\}_{p \geq 1} \in \mathcal{S}_{\infty}$.

Proof. Note that

$$
c_{1}\left(L^{p}, h_{p}\right)=\left(p-n_{p}\right) c_{1}(L, h)+n_{p} c_{1}\left(L, h_{0}\right) \geq \varepsilon n_{p} \omega, \frac{A_{p}}{p}=\left\|c_{1}(L, h)\right\|=\left\|c_{1}\left(L, h_{0}\right)\right\|>0 .
$$

Hence

$$
c_{1}\left(L^{p}, h_{p}\right) \leq p\left(c_{1}(L, h)+c_{1}\left(L, h_{0}\right)\right)=A_{p} T_{0}, \text { with } T_{0}=\frac{1}{\left\|c_{1}(L, h)\right\|}\left(c_{1}(L, h)+c_{1}\left(L, h_{0}\right)\right),
$$

and Theorems 1.1 and 4.1 apply in this setting and conclude the proof.

Corollary 5.1 applies to the case of the Satake-Baily-Borel compactifications of arithmetic quotients. Let $D$ be a bounded symmetric domain in $\mathbb{C}^{n}$ and let $\Gamma$ be a torsionfree arithmetic lattice. Then $U:=D / \Gamma$ is a smooth quasi-projective variety, called an arithmetic variety. The Satake-Borel-Baily compactification of $U$ is a normal compact analytic space $X$ containing $U$ as a Zariski open dense set and which is minimal with this property, that is, given any normal compactification $U \subset X^{\prime}$, the identity map on $U$ extends to a holomorphic map $X^{\prime} \rightarrow X$ (see Satake [Sa], Borel-Baily [BB] ). Moreover, $\operatorname{codim}(X \backslash U) \geq 2$.

The Bergman metric $\beta_{D}$ on $D$ descends to a complete Kähler metric $\beta_{U}$ on $U$, which is Kähler-Einstein with $\operatorname{Ric}_{\beta_{U}}=-\beta_{U}$. We denote by $h^{K_{U}}$ the Hermitian metric induced by $\beta$ on $K_{U}$. Then $c_{1}\left(K_{U}, h^{K_{U}}\right)=-\operatorname{Ric}_{\beta_{U}}=\beta_{U}$.

Lemma 5.3. Assume that $\Gamma$ is neat. There exists an ample line bundle $F \rightarrow X$ such that $\left.F\right|_{U}=K_{U}$ and a singular metric $h^{F}$ on $F$ such that $\left.h^{F}\right|_{U}=h^{K_{U}}$, such that $c_{1}\left(F, h^{F}\right)$ is a Kähler current on $X$ and $\left.c_{1}\left(F, h^{F}\right)\right|_{U}=\beta_{U}$. Hence the Bergman Kähler metric $\beta_{U}$ extends to a Kähler current $\beta_{X}$ on $X$.

Proof. The existence of an ample line bundle $F \rightarrow X$ such that $\left.F\right|_{U}=K_{U}$ is shown in [Mu, Proposition 3.4(b)]; the bundle $F$ is the bundle used in [BB, Theorem 10.11] to embed $X$ into a projective space. Since $c_{1}\left(K_{U}, h^{K_{U}}\right)=\beta_{U}$, the local weights of $h^{K_{U}}$ are psh, so they extend to $X$. Thus, the metric $h^{K_{U}}$ extends to a metric $h^{F}$ on $F \rightarrow X$ and $c_{1}\left(K_{U}, h^{K_{U}}\right)$ extends to a positive closed current $c_{1}\left(F, h^{F}\right)$ on $X$.

To see that $c_{1}\left(F, h^{F}\right)$ is a Kähler current, one can describe $F$ in the following way. By [AMRT], $U$ admits a smooth toroidal compactification $\tilde{X}$. In particular, $\Sigma:=\widetilde{X} \backslash U$ is a divisor with normal crossings. Let $L:=K_{\widetilde{X}} \otimes \mathscr{O}_{\widetilde{X}}(\Sigma)$. Then the metric $h^{K_{U}}$ defines a singular metric $h^{L}$ on $L$ such that $c_{1}\left(L, h^{L}\right)$ is a closed positive current on $\widetilde{X}$ which extends $\beta_{U} /(2 \pi)$, cf. [CM1, Lemma 6.8]. It follows from [Mu, Proposition 3.4] (see also [CM1, Section 6.4]) that $h^{K_{U}}$ is a good metric in the sense of Mumford, so that $\beta_{U}$ has Poincaré growth near $\Sigma$. Therefore, $c_{1}\left(L, h^{L}\right)$ is a Kähler current on $\widetilde{X}$. If $\tau: \widetilde{X} \rightarrow X$ 
is the unique holomorphic map extending the identity on $U$, we have $L=\tau^{*} F$. Hence $c_{1}\left(F, h^{F}\right)=\tau_{*} c_{1}\left(L, h^{L}\right)$, so $c_{1}\left(F, h^{F}\right)$ is a Kähler current on $X$.

Corollary 5.4. Let $X$ be the Satake-Baily-Borel compactification of a smooth arithmetic variety $U=D / \Gamma$, where $\Gamma$ is neat. Let $\left(F, h^{F}\right)$ be the extension of $\left(K^{U}, h^{K_{U}}\right)$ given by Lemma 5.3. where $h^{K_{U}}$ is induced by the Bergman metric $\beta_{U}$. Then the conclusions of Corollary 5.1] hold for $c_{1}\left(F, h^{F}\right)=\beta_{X}$.

Note that $H_{(2)}^{0}\left(X, F^{p}\right)=H_{(2)}^{0}\left(U, K_{U}^{p}\right)=H^{0}\left(\widetilde{X}, K_{\widetilde{X}}^{p} \otimes \mathscr{O}_{\widetilde{X}}(\Sigma)^{p-1}\right)=H^{0}\left(\widetilde{X}, L^{p} \otimes \mathscr{O}_{\widetilde{X}}(\Sigma)^{-1}\right)$ is the space of cusp forms of weight $p$, cf. [CM1, Lemma 6.11]. Hence Corollary [5.4 says that the Bergman kernel $P_{p}$ of the space of cusps forms satisfies $\frac{1}{p} \log P_{p} \rightarrow 0$ in $L^{1}\left(X, \omega^{n}\right)$, where $\omega$ is any smooth Kähler form on $X$. Moreover, the normalized zero-currents of random cusp forms distribute on $X$ to the extension $\beta_{X}$ of the Bergman metric $\beta_{U}$ on $U$.

We consider next a more general situation as above. Let $X$ be a projective variety of general type with only canonical singularities such that the canonical divisor $\mathcal{K}_{X}$ is an ample $\mathbb{Q}$-divisor. There exists an integer $\ell>0$ such that $\mathcal{K}_{X}^{\ell}$ is integral and the holomorphic line bundle $L=\mathscr{O}_{X}\left(\mathcal{K}_{X}^{\ell}\right) \rightarrow X$ admits a smooth Hermitian metric $h^{L}$ such that $c_{1}\left(L, h^{L}\right)=\omega$ is a smooth Kähler form on $X$. By [EGZ, Theorem 7.8] there exists a unique $\varphi \in P S H(X, \omega) \cap L^{\infty}(X)$ such that

$$
c_{1}\left(L, e^{-2 \varphi} h^{L}\right)=\omega+d d^{c} \varphi=\omega_{\varphi}
$$

is a singular Kähler-Einstein metric in the sense of [EGZ, Definition 7.3]. In particular, $\omega_{\varphi}$ is a closed positive current on $X$ and its restriction to $X_{\text {reg }}$ is a smooth Kähler-Einstein metric of negative curvature. Corollary 5.2 yields the following.

Corollary 5.5. Let $X$ be a projective variety of general type with only canonical singularities and let $\omega_{\varphi}$ be the singular Kähler-Einstein metric (29). Let $h_{p}$ be the metric on $L^{p}$ constructed as in (28) using a sequence $\left\{n_{p}\right\}_{p \geq 1}$ as in (27) and the metrics $h_{0}=h^{L}$ and $h=e^{-2 \varphi} h^{L}$ on L. Let $\gamma_{p}, \mathcal{S}^{p}$ be the Fubini-Study current and the unit sphere associated to the space $H_{(2)}^{0}\left(X, L^{p}, h_{p}\right)$. Then $\frac{1}{p} \gamma_{p} \rightarrow \omega_{\varphi}, \frac{1}{p}\left[\sigma_{p}=0\right] \rightarrow \omega_{\varphi}$ as $p \rightarrow \infty$ weakly on $X$, for $\lambda_{\infty}$-a.e. sequence $\left\{\sigma_{p}\right\}_{p \geq 1} \in \mathcal{S}_{\infty}$.

A similar discussion applies to canonically polarized klt compact Kähler pairs $(X, \Delta)$ considered in [EGZ, Theorem 7.12] or to $\mathbb{Q}$-Fano Kähler spaces (that is, klt compact Kähler spaces with $-\mathcal{K}_{X}$ ample $\mathbb{Q}$-divisor).

5.2. Powers of ample line bundles. We specialize in the sequel the results of the previous corollary to the case when $(X, \omega)$ is a projective manifold with a polarization $\left(L, h_{0}\right)$, where $L$ is a positive line bundle on $X$ endowed with a smooth Hermitian metric $h_{0}$ such that $c_{1}\left(L, h_{0}\right)=\omega$. The set of singular Hermitian metrics $h$ on $L$ with $c_{1}(L, h) \geq 0$ is in one-to-one correspondence to the set $\operatorname{PSH}(X, \omega)$ of $\omega$-plurisubharmonic ( $\omega$-psh) functions on $X$, by associating to $\varphi \in P S H(X, \omega)$ the metric $h_{\varphi}=e^{-2 \varphi} h_{0}$ (see e.g., [D4, GZ1]). Note that

$$
c_{1}\left(L, h_{\varphi}\right)=\omega+d d^{c} \varphi=: \omega_{\varphi} .
$$


Corollary 5.6. Let $(X, \omega)$ be a compact Kähler manifold and $\left(L, h_{0}\right)$ be a positive line bundle on $X$ with $c_{1}\left(L, h_{0}\right)=\omega$. Let $\varphi \in P S H(X, \omega)$ and $h_{p}$ be a metric on $L^{p}$ constructed as in (28) using a sequence $\left\{n_{p}\right\}_{p \geq 1}$ as in (27) and the metrics $h_{0}$ and $h=h_{\varphi}$ on L. Let $\gamma_{p}$, $\mathcal{S}^{p}$ be the Fubini-Study current and the unit sphere associated to the space $H_{(2)}^{0}\left(X, L^{p}, h_{p}\right)$. Then $\frac{1}{p} \gamma_{p} \rightarrow \omega_{\varphi}, \frac{1}{p}\left[\sigma_{p}=0\right] \rightarrow \omega_{\varphi}$ as $p \rightarrow \infty$ weakly on $X$, for $\lambda_{\infty}$-a.e. sequence $\left\{\sigma_{p}\right\}_{p \geq 1} \in \mathcal{S}_{\infty}$. Moreover, if $\varphi$ is continuous then

$$
\frac{1}{p^{k}} \gamma_{p}^{k} \rightarrow \omega_{\varphi}^{k}=\left(\omega+d d^{c} \varphi\right)^{k} \text { weakly on } X, \text { for } k \leq n .
$$

Proof. The first conclusion follows directly from Corollary 5.2. If $\varphi$ is continuous and $P_{p}$ is the Bergman kernel function of the space $H_{(2)}^{0}\left(X, L^{p}, h_{p}\right)$ one can proceed as in the proof of [CM1, Theorem 5.3] to show that $\frac{1}{p} \log P_{p} \rightarrow 0$ uniformly on $X$. This implies the second conclusion of the corollary, as in [CM1, Theorem 5.4].

Note that since $h_{0}$ is smooth we have that $H_{(2)}^{0}\left(X, L^{p}, h^{p}\right) \subset H_{(2)}^{0}\left(X, L^{p}, h_{p}\right)$. Moreover, if the metric $h=h_{\varphi}$ is bounded (i.e., $\varphi$ is bounded) then equality holds, $H_{(2)}^{0}\left(X, L^{p}, h^{p}\right)=$ $H_{(2)}^{0}\left(X, L^{p}, h_{p}\right)=H^{0}\left(X, L^{p}\right)$.

We remark now that, instead of working with random sections of spheres, one can identify $H_{(2)}^{0}\left(X, L_{p}\right)$ to $\mathbb{C}^{d_{p}}$ by

$$
a=\left(a_{1}, \ldots, a_{d_{p}}\right) \in \mathbb{C}^{d_{p}} \longmapsto S_{a}=\sum_{j=1}^{d_{p}} a_{j} S_{j}^{p} \in H_{(2)}^{0}\left(X, L_{p}\right),
$$

and one can consider $a_{j}, 1 \leq j \leq d_{p}$, as independent identically distributed Gaussian random variables on $\mathbb{C}$. Thus the probability space $\left(\mathcal{S}^{p}, \lambda_{p}\right)$ is replaced by $\left(H_{(2)}^{0}\left(X, L_{p}\right), \mu_{p}\right)$, where

$$
d \mu_{p}(z)=\pi^{-d_{p}} e^{-\left(\left|z_{1}\right|^{2}+\ldots+\left|z_{d_{p}}\right|^{2}\right)} d m(z),
$$

and $d m(z)$ is the Lebesgue measure on $\mathbb{C}^{d_{p}}$. Let $\mu_{\infty}=\prod_{p=1}^{\infty} \mu_{p}$ be the product measure on the space $\mathcal{H}=\prod_{p=1}^{\infty} H_{(2)}^{0}\left(X, L_{p}\right)$. Since the measure $\mu_{p}$ is unitary invariant, Theorems 4.1 and 4.2 hold in this context with similar proofs. More precisely, we have the following:

Theorem 5.7. Let $(X, \omega),\left(L_{p}, h_{p}\right), p \geq 1$, verify assumptions $(A)$-(B).

(i) If $\sum_{p=1}^{\infty} \frac{1}{A_{p}^{2}}<\infty$ then for $\mu_{\infty}$-a.e. sequence $\left\{\sigma_{p}\right\}_{p \geq 1} \in \mathcal{H}$ we have that

$$
\lim _{p \rightarrow \infty} \frac{1}{A_{p}}\left(\left[\sigma_{p}=0\right]-c_{1}\left(L_{p}, h_{p}\right)\right)=0 \text {, in the weak sense of currents on } X .
$$

(ii) If condition (26) holds then there exists an increasing sequence of natural numbers $\left\{p_{j}\right\}_{j \geq 1}$ such that for $\mu_{\infty}$-a.e. sequence $\left\{\sigma_{p}\right\}_{p \geq 1} \in \mathcal{H}$ we have

$$
\lim _{j \rightarrow \infty} \frac{\left.\log \left|\sigma_{p_{j}}\right|\right|_{h_{j}}}{A_{p_{j}}}=0 \text { in } L^{1}\left(X, \omega^{n}\right) .
$$

Hence Corollary [5.6 can be seen as a generalization of Theorem 5.2 in [BL] which deals with the special case when $\varphi=\mathcal{V}_{K, q}^{*}$ is the weighted $\omega$-psh global extremal function of 
a compact $K \subset X$. Using different methods and a different inner product on $H^{0}\left(X, L^{p}\right)$ (note that $\mathcal{V}_{K, q}^{*}$ is bounded) it is shown in [BL, Theorem 5.2] that

$$
\frac{1}{p}\left[\sigma_{p}=0\right] \rightarrow \omega+d d^{c} \mathcal{V}_{K, q}^{*} \text { for } \mu_{\infty} \text {-a. e. sequences }\left\{\sigma_{p}\right\}_{p \geq 1} \in \prod_{p=1}^{\infty} H^{0}\left(X, L^{p}\right) .
$$

On the other hand [BL, Theorem 5.2] holds for more general probability measures than $\mu_{\infty}$ (see [BL, (2.1) and (2.2)]).

Remark 5.8. A particularly interesting case is when $X=\mathbb{P}^{n}, L=\mathcal{O}(1)$ and $\omega=\omega_{\mathrm{FS}}$ is the Fubini-Study metric in Corollary 5.6. In this case the class $P S H\left(\mathbb{P}^{n}, \omega_{\mathrm{FS}}\right)$ is in one-to-one correspondence with the Lelong class of psh functions on $\mathbb{C}^{n}$ of logarithmic growth, and the sections in $H^{0}\left(\mathbb{P}^{n}, \mathcal{O}(p)\right)$ can be identified to polynomials of degree $\leq p$ on $\mathbb{C}^{n}$ (see e.g., [BL, Section 5]). Therefore Corollary 5.6 yields a general equidistribution result for the zeros of a random sequence of polynomials on $\mathbb{C}^{n}$. For related results see [Ba], [BB] and references therein.

We consider now approximation of metrics with conic singularities [Do, T2]. Let $X$ be a Fano manifold, that is, the anti-canonical line bundle $K_{X}^{-1}$ is ample. Fix a Hermitian metric $h_{0}$ on $K_{X}^{-1}$, such that $\omega:=c_{1}\left(K_{X}^{-1}, h_{0}\right)$ is a Kähler metric. Recall that Hermitian metrics on $K_{X}^{-1}$ can be identified to volume forms on $X$. Let $D$ be a smooth divisor in the linear system defined by $K_{X}^{-\ell}$, so there exists a section $s \in H^{0}\left(X, K_{X}^{-\ell}\right)$ with $D=\operatorname{Div}(s)$. We fix a smooth metric $h$ on the bundle $\mathscr{O}_{X}(D)$. Let $\beta \in[0,1)$.

A Kähler metric $\widehat{\omega}$ on $X$ with cone angle $2 \pi(1-\beta)$ along $D$ is a current $\widehat{\omega} \in c_{1}(X)$ such that $\widehat{\omega}=\omega_{\varphi}=\omega+d d^{c} \varphi$ where $\varphi=\psi+|s|_{h}^{2-2 \beta} \in P S H(X, \omega)$ and $\psi \in \mathscr{C}^{\infty}(X) \cap P S H(X, \omega)$. In a neigbourhood of a point of $D$ where $D$ is given by $z_{1}=0$ the metric $\widehat{\omega}$ is equivalent to the cone metric $\frac{i}{2}\left(\left|z_{1}\right|^{-2 \beta} d z_{1} \wedge d \bar{z}_{1}+\sum_{j=2}^{n} d z_{j} \wedge d \bar{z}_{j}\right)$. The Riemannian metric determined by $\widehat{\omega}$ has conic singularity along $D$ of conic angle $2 \pi(1-\beta)$.

The metric $\widehat{\omega}$ defines a singular metric $h_{\widehat{\omega}}$ on $K_{X}^{-1}$ with curvature current $\operatorname{Ric}_{\widehat{\omega}}:=$ $c_{1}\left(K_{X}^{-1}, h_{\widehat{\omega}}\right)$. The metric $\widehat{\omega}$ is called Kähler-Einstein with conic singularities of cone angle $2 \pi(1-\beta)$ along $D$ if $\operatorname{Ric}_{\widehat{\omega}}=(1-\ell \beta) \widehat{\omega}+\beta[D]$, where $[D]$ is the current of integration on D.

Corollary 5.9. Let $X$ be a Fano manifold and let $\widehat{\omega}$ be a Kähler metric with cone angle $\beta$. Let $h_{p}$ be the metric on $K_{X}^{-p}$ constructed as in Corollary 5.2 using the metric $h=h_{\widehat{\omega}}$ on $K_{X}^{-1}$. Let $\gamma_{p}, \mathcal{S}^{p}$ be the Fubini-Study current and the unit sphere associated to the space $H_{(2)}^{0}\left(X, K_{X}^{-p}, h_{p}\right)$. Then $\frac{1}{p} \gamma_{p} \rightarrow \operatorname{Ric}_{\widehat{\omega}}$ and $\frac{1}{p}\left[\sigma_{p}=0\right] \rightarrow \operatorname{Ric}_{\widehat{\omega}}$ as $p \rightarrow \infty$ weakly on $X$, for $\lambda_{\infty}$-a.e. sequence $\left\{\sigma_{p}\right\}_{p \geq 1} \in \mathcal{S}_{\infty}$. If $\widehat{\omega}$ is a Kähler current, then we can take $h_{p}=h_{\widehat{\omega}}^{p}$ above.

Remark 5.10. If $\widehat{\omega}$ is a Kähler current, [HsM, Theorem 1.8] shows that the Bergman kernel of $H_{(2)}^{0}\left(X, K_{X}^{-p}, h_{\widehat{\omega}}^{p}\right)$ has a full asymptotic expansion in powers $p^{n-j}, j=0,1, \ldots$, and the Fubini-Study forms converge to $\operatorname{Ric}_{\widehat{\omega}}$ in the $\mathscr{C}^{\infty}$-topology on compact sets of $X \backslash D$.

5.3. Tensor products of powers of several line bundles. Another typical application of Theorem 1.1 is the following. 
Corollary 5.11. Let $(X, \omega)$ be a compact normal Kähler space. Assume that $\left(F_{j}, h^{F_{j}}\right)$, $1 \leq j \leq k$, are singular Hermitian holomorphic line bundles with $c_{1}\left(F_{j}, h^{F_{j}}\right) \geq 0$ and $c_{1}\left(F_{1}, h^{F_{1}}\right) \geq \varepsilon \omega$, for some $\varepsilon>0$. Let $T=\sum_{j=1}^{k} r_{j} c_{1}\left(F_{j}, h^{F_{j}}\right)$, where $r_{j} \geq 0$, and let $\left\{m_{j, p}\right\}_{p}, 1 \leq j \leq k$, be sequences of natural numbers such that

$$
m_{1, p} \rightarrow \infty, \frac{m_{j, p}}{p} \rightarrow r_{j}, 1 \leq j \leq k, \text { as } p \rightarrow \infty .
$$

Let $P_{p}, \gamma_{p}, \mathcal{S}^{p}$ be the Bergman kernel function, Fubini-Study current, and respectively the unit sphere, associated to $H_{(2)}^{0}\left(X, L_{p}\right)$, where

$$
L_{p}=F_{1}^{m_{1, p}} \otimes \ldots \otimes F_{k}^{m_{k, p}}, h_{p}=\left(h^{F_{1}}\right)^{m_{1, p}} \otimes \ldots \otimes\left(h^{F_{k}}\right)^{m_{k, p}} .
$$

Then, as $p \rightarrow \infty$, we have $\frac{1}{p} \log P_{p} \rightarrow 0$ in $L^{1}\left(X, \omega^{n}\right), \frac{1}{p} \gamma_{p} \rightarrow T$ and $\frac{1}{p}\left[\sigma_{p}=0\right] \rightarrow T$ in the weak sense of currents on $X$, for $\lambda_{\infty}$-a.e. sequence $\left\{\sigma_{p}\right\}_{p \geq 1} \in \mathcal{S}_{\infty}$.

Proof. We may assume that $m_{j, p} / p<r_{j}+1$ for all $1 \leq j \leq k, p \geq 1$, so

$$
\begin{aligned}
& c_{1}\left(L_{p}, h_{p}\right)=\sum_{j=1}^{k} m_{j, p} c_{1}\left(F_{j}, h^{F_{j}}\right) \geq \varepsilon m_{1, p} \omega, \\
& c_{1}\left(L_{p}, h_{p}\right) \leq p T_{0}, \text { where } T_{0}=\sum_{j=1}^{k}\left(r_{j}+1\right) c_{1}\left(F_{j}, h^{F_{j}}\right) .
\end{aligned}
$$

Moreover $c_{1}\left(L_{p}, h_{p}\right) / p \rightarrow T$ as $p \rightarrow \infty$. Note that

$$
\frac{A_{p}}{p}=\frac{1}{p} \sum_{j=1}^{k} m_{j, p}\left\|c_{1}\left(F_{j}, h^{F_{j}}\right)\right\| \rightarrow \sum_{j=1}^{k} r_{j}\left\|c_{1}\left(F_{j}, h^{F_{j}}\right)\right\| .
$$

The conclusion follows from Theorems 1.1 and 4.1 , since

$$
\frac{\gamma_{p}}{p}=\frac{A_{p}}{p} \frac{1}{A_{p}}\left(\gamma_{p}-c_{1}\left(L_{p}, h_{p}\right)\right)+\frac{1}{p} c_{1}\left(L_{p}, h_{p}\right) .
$$

The fact that $\frac{1}{p}\left[\sigma_{p}=0\right] \rightarrow T$ weakly on $X$, for $\lambda_{\infty}$-a.e. sequence $\left\{\sigma_{p}\right\}_{p \geq 1} \in \mathcal{S}_{\infty}$, follows as in the proof of Theorem 4.1, where we normalize all currents dividing by $p$, by using that $A_{p} \lesssim p$ and $\sum_{p=1}^{\infty} p^{-2}$ is finite.

Let us remark that Theorem 4.2 holds in the context of Corollaries 5.1, 5.2, 5.6, 5.11, since the condition (26) holds in all of these situations.

5.4. Covering manifolds. Let $(X, \omega)$ be a compact Kähler manifold of dimension $n$ and $\left(L_{p}, h_{p}\right), p \geq 1$, be a sequence of singular Hermitian holomorphic line bundles on $X$ satisfying condition (11). Recall that $A_{p}=\int_{X} c_{1}\left(L_{p}, h_{p}\right) \wedge \omega^{n-1}$. Let $q: \widetilde{X} \rightarrow X$ be a (paracompact) Galois covering of $X$, where $q$ is the canonical projection. Let us denote by $\widetilde{\omega}=q^{*} \omega, \widetilde{L}_{p}=q^{*}\left(L_{p}\right)$ and by $\widetilde{h}_{p}$ the metric on $\widetilde{L}_{p}$ which is the pull-back of the metric $h_{p}$. We let $H_{(2)}^{0}\left(\widetilde{X}, \widetilde{L}_{p}\right)$ be the Bergman space of $L^{2}$-holomorphic sections of $\widetilde{L}_{p}$ relative to the metric $\widetilde{h}_{p}$ and the volume form $\widetilde{\omega}^{n} / n$ ! on $\widetilde{X}$, defined as in (3), endowed with the obvious 
inner product. We define the Bergman kernel function $\widetilde{P}_{p}$ and Fubini-Study currents $\widetilde{\gamma}_{p}$ associated to $H_{(2)}^{0}\left(\widetilde{X}, \widetilde{L}_{p}\right)$ as in (4). In this context, $d_{p} \in \mathbb{N} \cup\{\infty\}$, and these objects are well defined even for $d_{p}=\infty$, see [CM1, Lemmas 3.1-3.2].

Note that $\widetilde{\omega}$ is a complete Kähler metric on $\widetilde{X}$ and $c_{1}\left(\widetilde{L}_{p}, \widetilde{h}_{p}\right)=q^{*} c_{1}\left(L_{p}, h_{p}\right) \geq a_{p} \widetilde{\omega}$. Since $\operatorname{Ric}_{\omega} \geq-B \omega$ holds on $X$, for some constant $B>0$, and $q$ is a local biholomorphism, we have $\operatorname{Ric}_{\tilde{\omega}} \geq-B \widetilde{\omega}$ on $\widetilde{X}$. Moreover, if $K \Subset \widetilde{X}$ there exists a constant $C_{K}>0$ such that

$$
\int_{K} c_{1}\left(\widetilde{L}_{p}, \widetilde{h}_{p}\right) \wedge \widetilde{\omega}^{n-1} \leq C_{K} A_{p}
$$

A straightforward adaptation of the proofs given above yields:

$$
\begin{aligned}
& \frac{1}{A_{p}} \log \widetilde{P}_{p} \rightarrow 0 \text { in } L_{l o c}^{1}\left(\widetilde{X}, \widetilde{\omega}^{n}\right), \\
& \frac{1}{A_{p}}\left(\widetilde{\gamma}_{p}-c_{1}\left(\widetilde{L}_{p}, \widetilde{h}_{p}\right)\right) \rightarrow 0 \text { weakly on } \widetilde{X} .
\end{aligned}
$$

Assume moreover that the metrics $h_{p}$ are of class $\mathscr{C}^{3}$ and condition (5) is fulfilled, that is, $\varepsilon_{p}:=\left\|h_{p}\right\|_{3}^{1 / 3} a_{p}^{-1 / 2} \rightarrow 0$ as $p \rightarrow \infty$. Then there exists $p_{0} \in \mathbb{N}$ such that for all $p>p_{0}$

$$
\left|\widetilde{P}_{p} \frac{\widetilde{\omega}^{n}}{c_{1}\left(\widetilde{L}_{p}, \widetilde{h}_{p}\right)^{n}}-1\right| \leq C \varepsilon_{p}^{2 / 3} \text { on } \tilde{X},
$$

where $C>0$ is a constant depending only on $(X, \omega)$. Estimates (6) and (31) show that the asymptotics of the Bergman kernels $\widetilde{P}_{p}(\widetilde{x})$ and $P_{p}(x), x=q(\widetilde{x})$, are the same. For $\left(L_{p}, h_{p}\right)=\left(L^{p}, h^{p}\right)$ with a smooth metric $h$ satisfying $c_{1}(L, h) \geq a \omega$ this follows from [MM1, Theorem 6.1.4].

\section{REFERENCES}

[A] A. Andreotti, Théorèmes de dépendance algébrique sur les espaces complexes pseudo-concaves, Bull. Soc. Math. France 91 (1963), 1-38.

[AMRT] A. Ash, D. Mumford, M. Rapoport and Y. S. Tai, Smooth compactifications of locally symmetric varieties, 2nd ed. With the collaboration of Peter Scholze. Cambridge Mathematical Library. Cambridge University Press, Cambridge, 2010.

[BB] W. L. Baily and A. Borel, Compactifcation of arithmetic quotients of bounded symmetric domains, Ann. Math. 84 (1966), 442-528.

[Ba] T. Bayraktar, Equidistribution of zeros of random holomorphic sections, arXiv:1312.0933.

[Be] B. Berndtsson, Bergman kernels related to Hermitian line bundles over compact complex manifolds, Explorations in complex and Riemannian geometry, Contemp. Math. 332 2003, 1-17.

[BM] E. Bierstone and P. Milman, Canonical desingularization in characteristic zero by blowing up the maximum strata of a local invariant, Invent. Math. 128 (1997), 207-302.

[BL] T. Bloom and N. Levenberg, Random polynomials and pluripotential-theoretic extremal functions, Potential Anal. 42 (2015), no. 2, 311-334.

[Bo] S. Boucksom, On the volume of a line bundle, Internat. J. Math. 13 (2002), 1043-1063.

[Ca] D. Catlin, The Bergman kernel and a theorem of Tian, in Analysis and geometry in several complex variables (Katata, 1997), 1-23, Trends Math., Birkhäuser, Boston, 1999.

[CM1] D. Coman and G. Marinescu, Equidistribution results for singular metrics on line bundles, Ann. Sci. École Norm. Supér. (4) 48 (2015), 497-536. 
[CM2] D. Coman and G. Marinescu, Convergence of Fubini-Study currents for orbifold line bundles, Internat. J. Math. 24 (2013), 1350051, 27 pp.

[CM3] D. Coman and G. Marinescu, On the approximation of positive closed currents on compact Kähler manifolds, Math. Rep. (Bucur.) 15(65) (2013), 373-386.

[DLM] X. Dai, K. Liu, and X. Ma, On the asymptotic expansion of Bergman kernel, J. Differential Geom. 72 (2006), 1-41.

[D1] J.-P. Demailly, Courants positifs extrêmaux et conjecture de Hodge, Invent. Math. 69 (1982), 347374.

[D2] J.-P. Demailly, Estimations $L^{2}$ pour l'opérateur $\bar{\partial}$ d'un fibré holomorphe semipositif au-dessus d'une variété kählérienne complète, Ann. Sci. École Norm. Sup. 15 (1982), 457-511.

[D3] J.-P. Demailly, Mesures de Monge-Ampère et caractérisation géométrique des variétés algébriques affines, Mém. Soc. Math. France (N.S.) No. 19 (1985), 1-125.

[D4] J.-P. Demailly, Singular Hermitian metrics on positive line bundles, in Complex algebraic varieties (Bayreuth, 1990), Lecture Notes in Math. 1507, Springer, Berlin, 1992, 87-104.

[D5] J.-P. Demailly, Regularization of closed positive currents and intersection theory, J. Algebraic Geom. 1 (1992), 361-409.

[D7] J.-P. Demailly, A numerical criterion for very ample line bundles, J. Differential Geom. 37 (1993), 323-374.

[DMS] T.-C. Dinh, G. Marinescu and V. Schmidt, Asymptotic distribution of zeros of holomorphic sections in the non compact setting, J. Stat. Phys. 148 (2012), 113-136.

[DS1] T.-C. Dinh and N. Sibony, Distribution des valeurs de transformations méromorphes et applications, Comment. Math. Helv. 81 (2006), 221-258.

[DS2] T.-C. Dinh and N. Sibony, Dynamics in several complex variables: endomorphisms of projective spaces and polynomial-like mappings, Holomorphic dynamical systems, 165-294, Lecture Notes in Math. 1998, Springer, Berlin, 2010.

[DNS] T.-C. Dinh, V.-A. Nguyên, and N. Sibony, Dynamics of horizontal-like maps in higher dimension, Adv. Math. 219 (2008), 1689-1721.

[Do] S. K. Donaldson, Kähler metrics with cone singularities along a divisor, Essays in mathematics and its applications, Springer, Heidelberg, 2012, 49-79.

[EGZ] P. Eyssidieux, V. Guedj and A. Zeriahi, Singular Kähler-Einstein metrics, J. Amer. Math. Soc. 22 (2009), 607-639.

[FN] J. E. Fornæss and R. Narasimhan, The Levi problem on complex spaces with singularities, Math. Ann. 248 (1980), 47-72.

[FS] J. E. Fornæss and N. Sibony, Oka's inequality for currents and applications, Math. Ann. 301 (1995), 399-419.

[GW] D. Gayet and J.-Y. Welschinger, What is the total Betti number of a random real hypersurface?, J. Reine Angew. Math. 689 (2014), 137-168.

[GM] C. Grant Melles and P. Milman, Classical Poincaré metric pulled back off singularities using a Chowtype theorem and desingularization, Ann. Fac. Sci. Toulouse Math. (6) 15 (2006), 689-771.

[Gr] H. Grauert, Über Modifikationen und exzeptionelle analytische Mengen, Math. Ann. 146 (1962), 331-368.

[GR1] H. Grauert and R. Remmert, Plurisubharmonische Funktionen in komplexen Räumen, Math. Z. 65 (1956), 175-194.

[GR2] H. Grauert and R. Remmert, Coherent Analytic Sheaves, Springer, Berlin, 1984. Grundlehren der Mathematischen Wissenschaften, 265, Springer-Verlag, Berlin, 249 pp., 1984.

[G] V. Guedj, Approximation of currents on complex manifolds, Math. Ann. 313 (1999), 437-474.

[GZ1] V. Guedj and A. Zeriahi, Intrinsic capacities on compact Kähler manifolds, J. Geom. Anal. 15 (2005), 607-639.

[H1] L. Hörmander, An introduction to complex analysis in several variables, Third edition, NorthHolland Mathematical Library, vol. 7, North-Holland Publishing Co., Amsterdam, 1990, xii+254. 
[H2] L. Hörmander, Notions of Convexity, Reprint of the 1994 edition, Modern Birkhäuser Classics, Basel, Birkhäuser, 2007, viii, 414 pp.

[HsM] C.-Y. Hsiao and G. Marinescu, Asymptotics of spectral function of lower energy forms and Bergman kernel of semi-positive and big line bundles, Comm. Anal. Geom. 22 (2014), 1-108.

[JS] S. Ji and B. Shiffman, Properties of compact complex manifolds carrying closed positive currents, J. Geom. Anal. 3 (1993), 37-61.

[KM] J. Kollár and T. Matsusaka, Riemann-Roch type inequalities, Amer. J. Math. 105 (1983), 229-252.

[MM1] X. Ma and G. Marinescu, Holomorphic Morse Inequalities and Bergman Kernels, Progress in Math., vol. 254, Birkhäuser, Basel, 2007, xiii, 422 pp.

[MM2] X. Ma and G. Marinescu, Generalized Bergman kernels on symplectic manifolds, Adv. Math. 217 (2008), 1756-1815.

[M] G. Marinescu, A criterion for Moishezon spaces with isolated singularities, Ann. Mat. Pura Appl. (4) 185 (2005), no. 1, 1-16.

[Moi] B. G. Moishezon, Reduction theorems for compact complex spaces with a sufficiently large field of meromorphic functions. (Russian), Izv. Akad. Nauk SSSR Ser. Mat. 31 (1967), 1385-1414. English translation: Resolution theorems for compact complex spaces with a sufficiently large field of meromorphic functions, Math. USSR - Izvestija 1 (1967), no. 6, 1331-1356.

[Mu] D. Mumford, Hirzebruch's proportionality theorem in the noncompact case, Invent. Math. 42 (1977), 239-272.

[N] R. Narasimhan, The Levi problem for complex spaces II, Math. Ann. 146 (1962), 195-216.

[NS] L. Nicolaescu and N. Savale, The Gauss-Bonnet-Chern theorem: a probabilistic perspective, arXiv:1404.5206.

[NV] S. Nonnenmacher and A. Voros, Chaotic eigenfunctions in phase space, J. Stat. Phys. 92 (1998), no. 3-4, 451-518.

[O] T. Ohsawa, Hodge spectral sequence and symmetry on compact Kähler spaces, Publ. Res. Inst. Math. Sci. 23 (1987), 613-625.

[OT] T. Ohsawa and K. Takegoshi, On the extension of $L^{2}$ holomorphic functions, Math. Z. 195 (1987), 197-204.

[Oka] K. Oka, Sur les fonctions des plusieurs variables. III: Deuxième problème de Cousin, J. Sc. Hiroshima Univ. 9 (1939), 7-19.

[R] R. Richberg, Stetige streng pseudokonvexe Funktionen, Math. Ann. 175 (1968), 256-286.

[R] W. D. Ruan, Canonical coordinates and Bergman metrics, Comm. Anal. Geom. 6 (1998), 589-631.

[Sa] I. Satake, On compactifications of the quotient spaces for arithmetically defined discontinuous groups, Ann. Math. 72 (1960), 555-580.

[Sh] B. Shiffman, Convergence of random zeros on complex manifolds, Sci. China Ser. A 51 (2008), 707-720.

[SZ1] B. Shiffman and S. Zelditch, Distribution of zeros of random and quantum chaotic sections of positive line bundles, Comm. Math. Phys. 200 (1999), 661-683.

[SZ2] B. Shiffman and S. Zelditch, Number variance of random zeros on complex manifolds, Geom. Funct. Anal. 18 (2008), 1422-1475.

[T1] G. Tian, On a set of polarized Kähler metrics on algebraic manifolds, J. Differential Geom. 32 (1990), 99-130.

[T2] G. Tian, Kähler-Einstein metrics on algebraic manifolds, Lecture notes in Mathematics, Volume 1646 (1996), 143-185.

[Z] S. Zelditch, Szegö kernels and a theorem of Tian, Internat. Math. Res. Notices 1998, no. 6, 317331. 
Department of Mathematics, Syracuse University, Syracuse, NY 13244-1150, USA

E-mail address: dcoman@syr.edu

Institut Universitaire de France \& Université Paris Diderot - Paris 7, UFR DE MAThÉmatiques, CASE 7012, 75205 PARIS CedeX 13, France

E-mail address: xiaonan.ma@imj-prg.fr

Universität ZU KÖln, Mathematisches Institut, Weyertal 86-90, 50931 Köln, Deutschland \& Institute of MATHEMATICS 'Simion STOILOW', Romanian ACADEMY, BUCHAREST, ROMANiA

E-mail address: gmarines@math. uni-koeln.de 